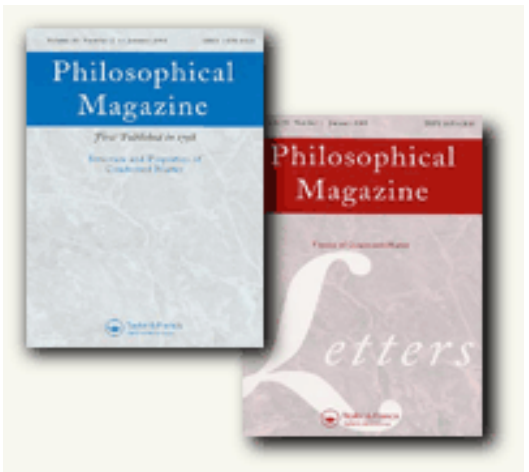

\title{
Slip planes and kink properties of screw dislocations in high-purity niobium
}

\begin{tabular}{|r|l|}
\hline Journal: & Philosophical Magazine \& Philosophical Magazine Letters \\
\hline Manuscript ID: & TPHM-05-Sep-0419 \\
\hline Journal Selection: & Philosophical Magazine \\
\hline Author: & $29-$ Sep-2005 \\
\hline Keywords: & $\begin{array}{l}\text { plasticity of metals, dislocation dynamics, refractory metals, } \\
\text { mechanical properties, deformation properties, deformation } \\
\text { mechanisms, internal friction }\end{array}$ \\
\hline Keywords (user supplied): & $\begin{array}{l}\text { Peierls barriers, body-centred cubic metals, kinks on screw } \\
\text { dislocations }\end{array}$ \\
\hline & $\begin{array}{l}\text { Seeger, Alfred; Max-Planck-Institut fuer Metallforschung } \\
\text { Holzwarth, Uwe; European Commission, Joint Research Centre }\end{array}$ \\
\hline & $\begin{array}{l}\text { Note: The following files were submitted by the author for peer review, but cannot be converted } \\
\text { to PDF. You must view these files (e.g. movies) online. }\end{array}$ \\
\hline SeegerHolzwarth.tex &
\end{tabular}

\section{S) ScholaroNE \\ Manuscript Central}


line-tension approximation

$$
\begin{gathered}
V_{\mathrm{kp}}^{\mathrm{LT}}=a b\left[\gamma_{\mathrm{d}} / U^{\prime \prime}(a)\right]^{1 / 2} \ln \left(\tilde{\sigma} / \sigma^{*}\right) \\
-\ldots----\sigma^{*}=\hat{\sigma}^{*}
\end{gathered}
$$

elastic-interaction approximation

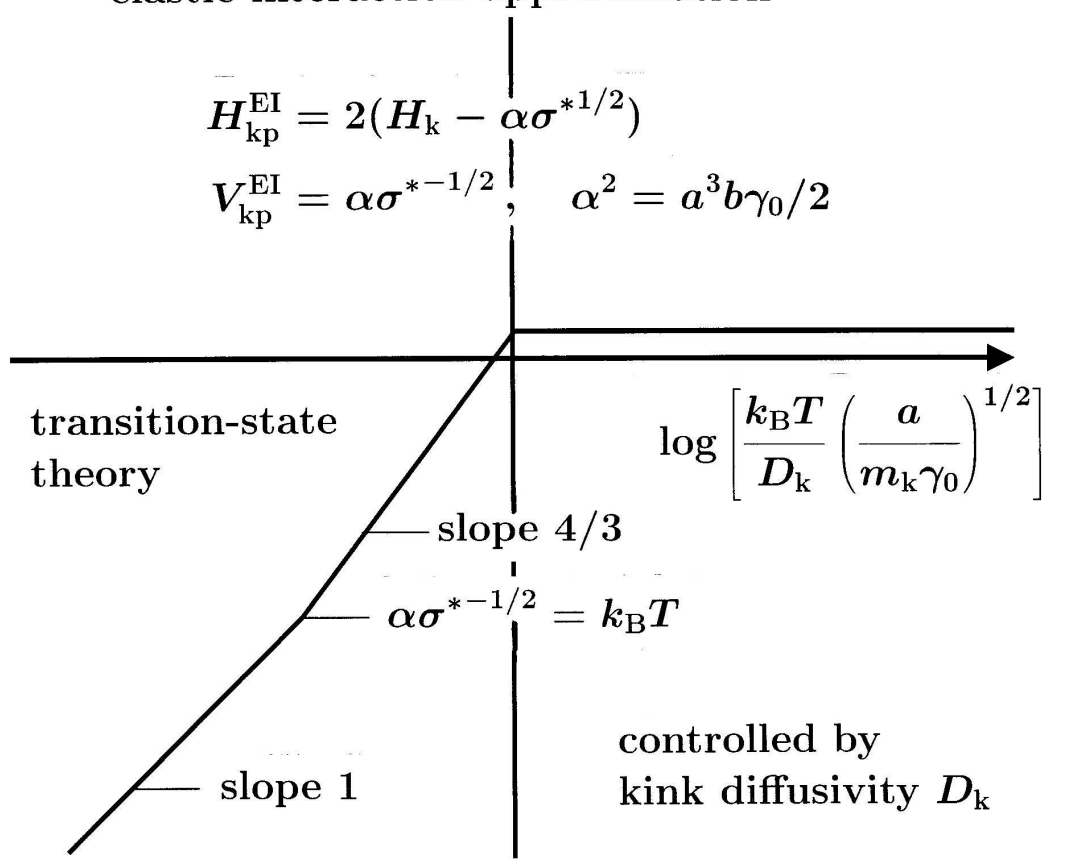

Seeger and Holzwarth Figure 1 $188 \times 232 \mathrm{~mm}(600 \times 600 \mathrm{DPI})$ 


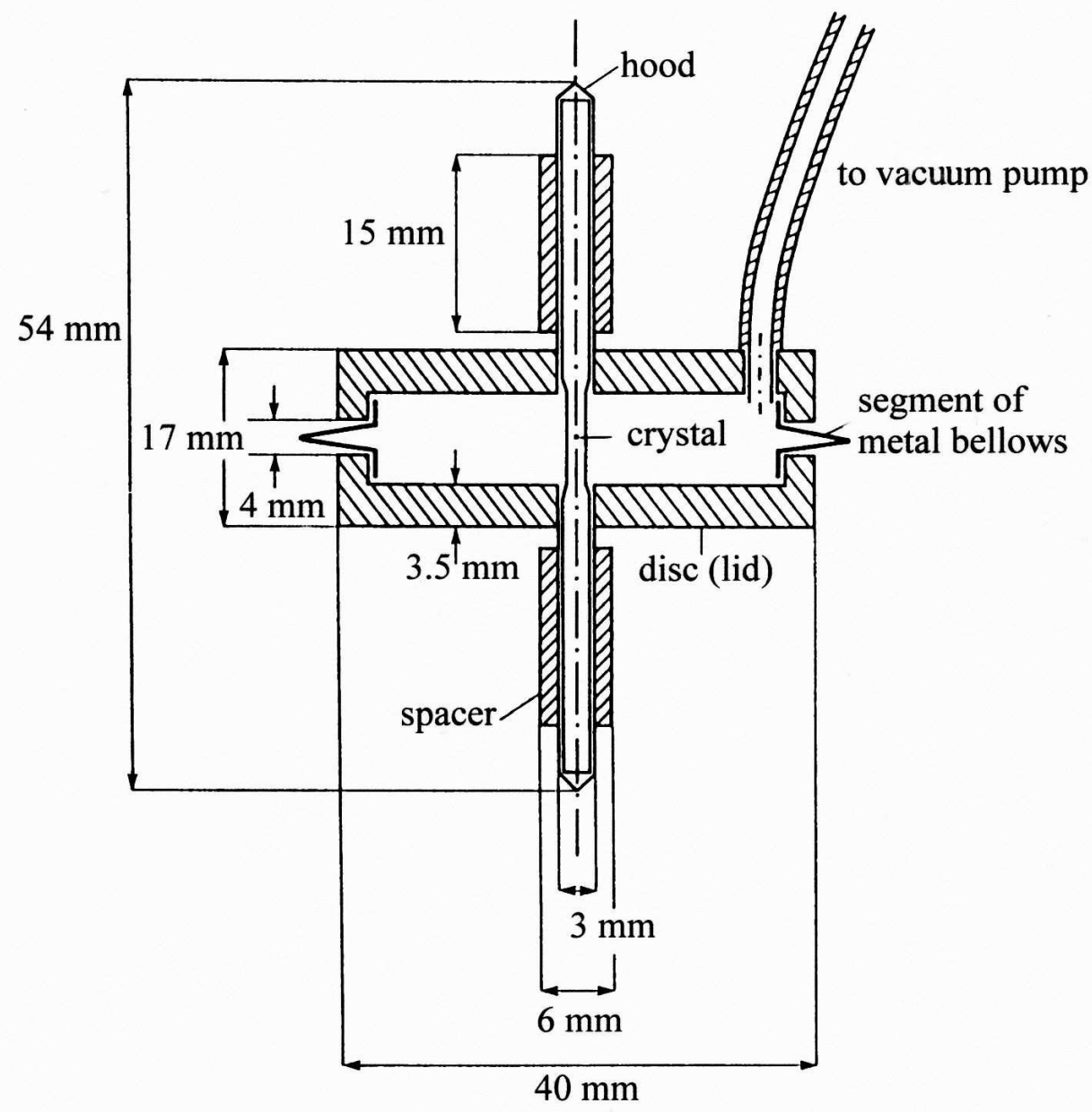

Seeger and Holzwarth Figure 2 $128 \times 133 \mathrm{~mm}(300 \times 300$ DPI) 


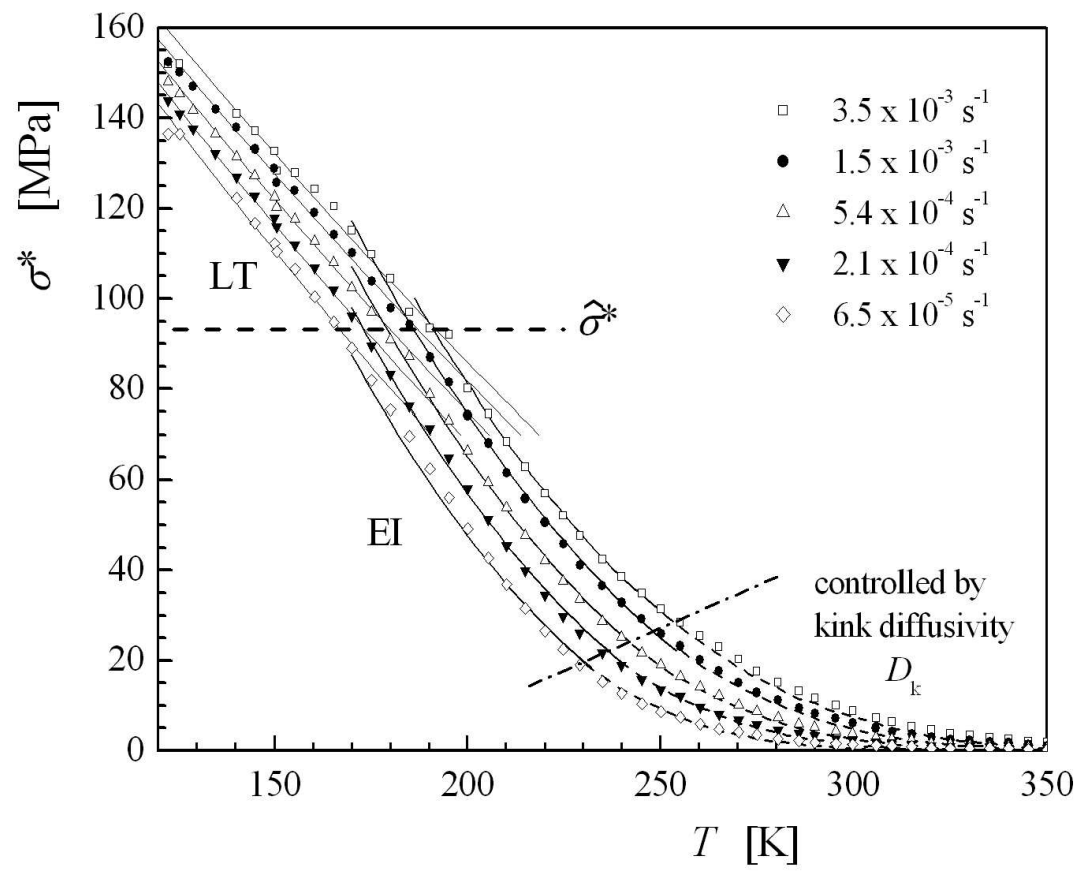

Seeger and Holzwarth Figure 3 $279 \times 215 \mathrm{~mm}(150 \times 150 \mathrm{DPI})$ 


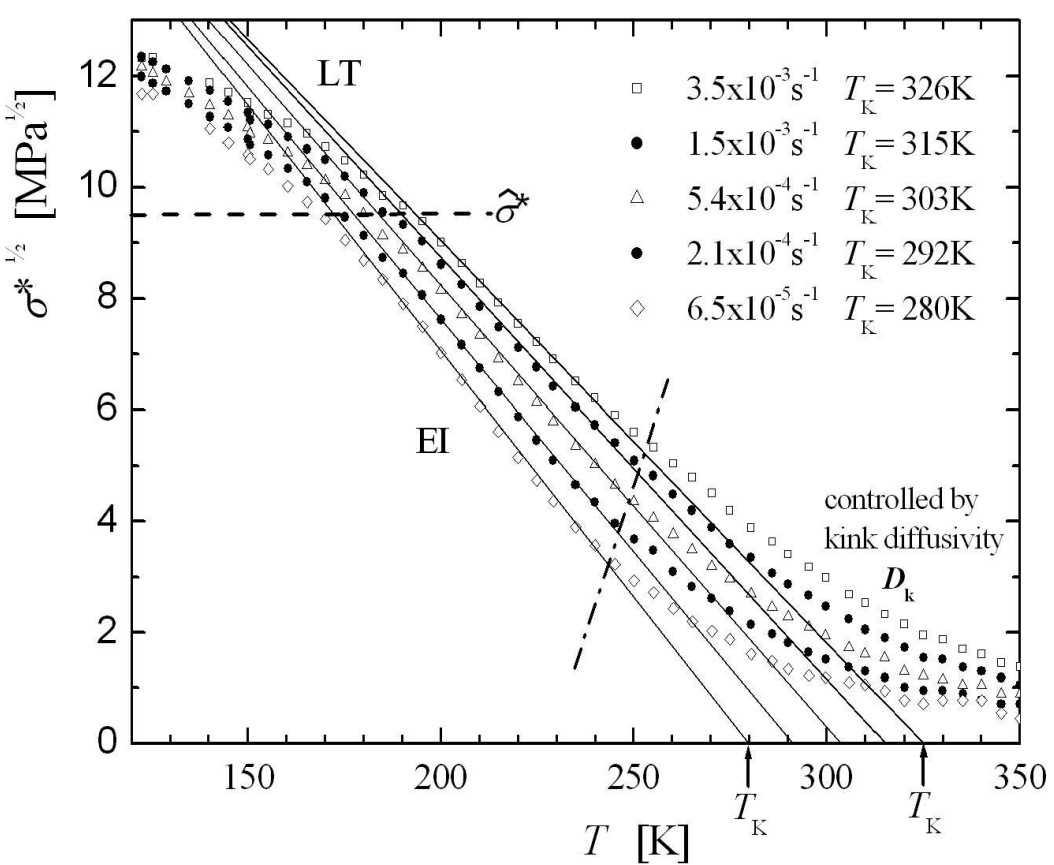

Seeger and Holzwarth Figure 4 $279 \times 215 \mathrm{~mm}(150 \times 150 \mathrm{DPI})$ 


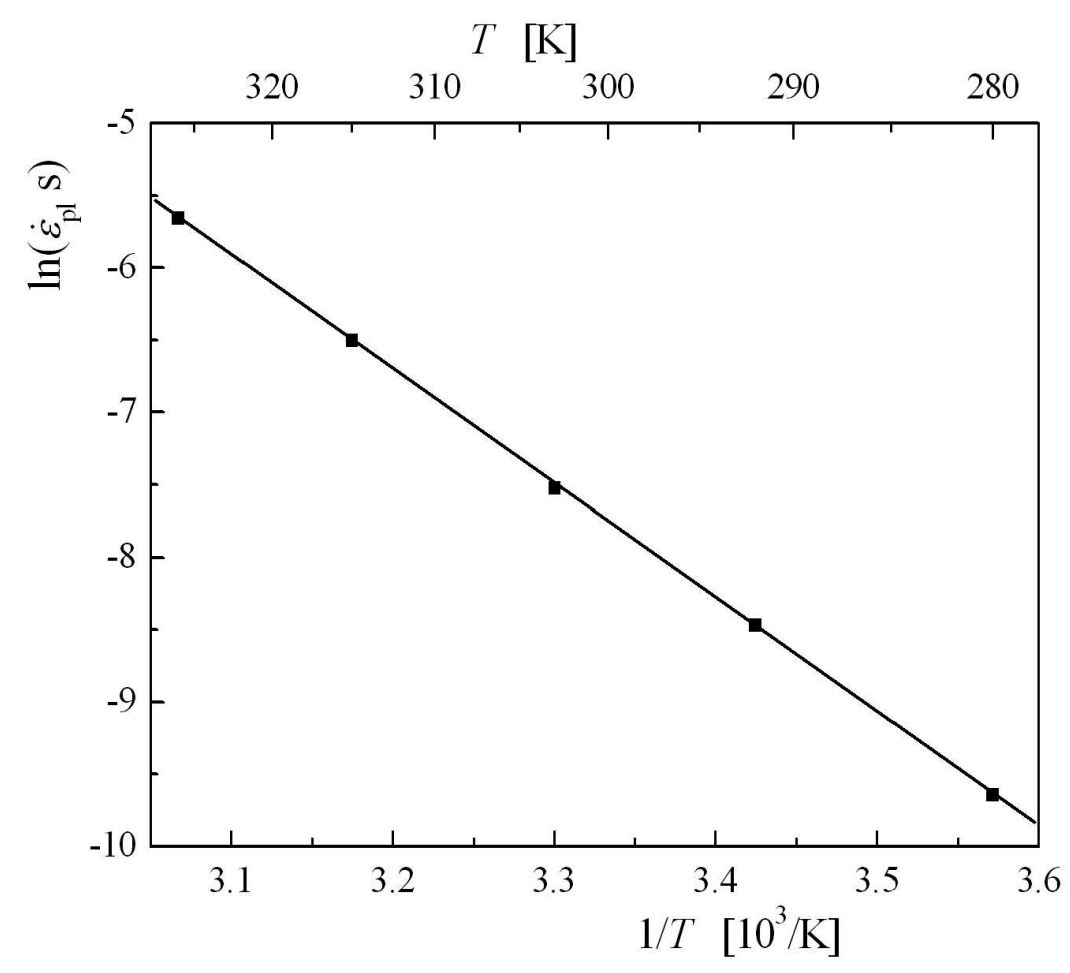

Seeger and Holzwarth Figure 5 $279 \times 215 \mathrm{~mm}(150 \times 150 \mathrm{DPI})$ 


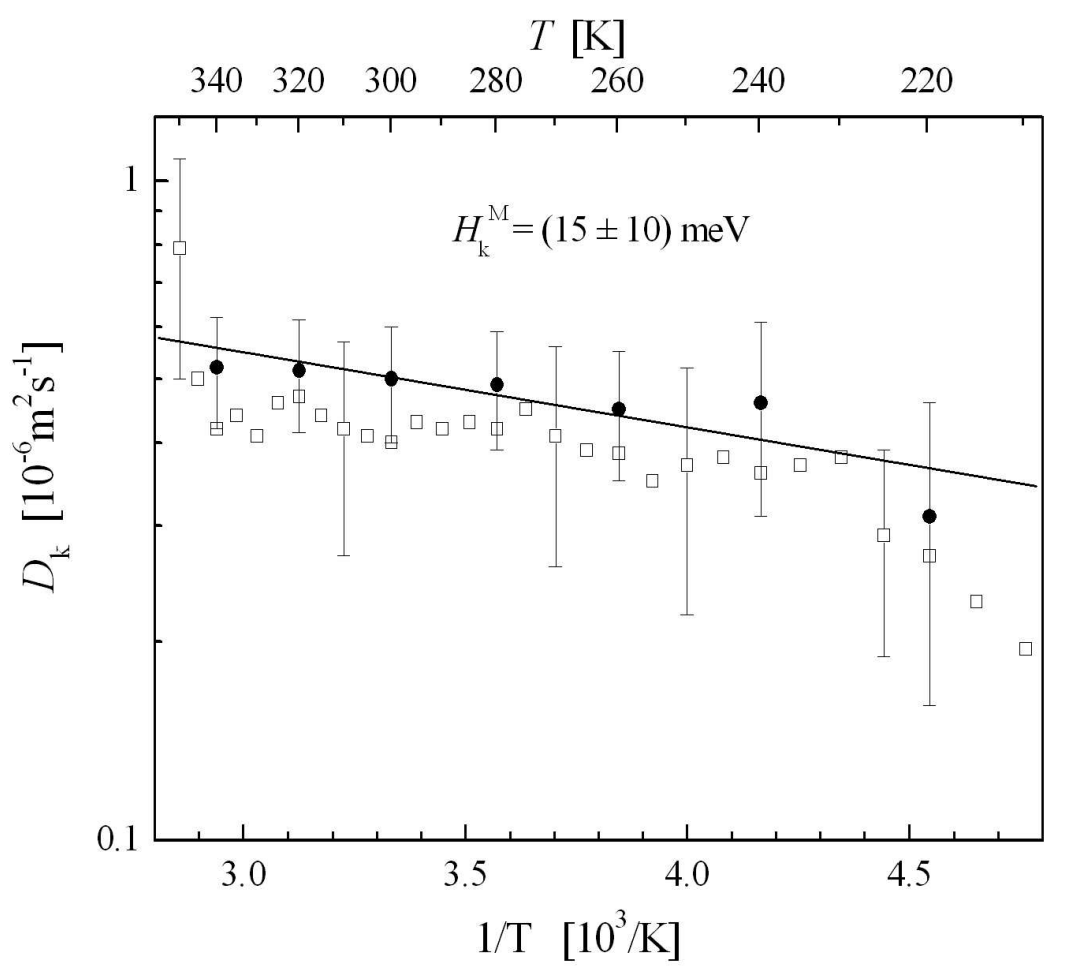

Seeger and Holzwarth Figure 6 $279 \times 215 \mathrm{~mm}(150 \times 150 \mathrm{DPI})$ 


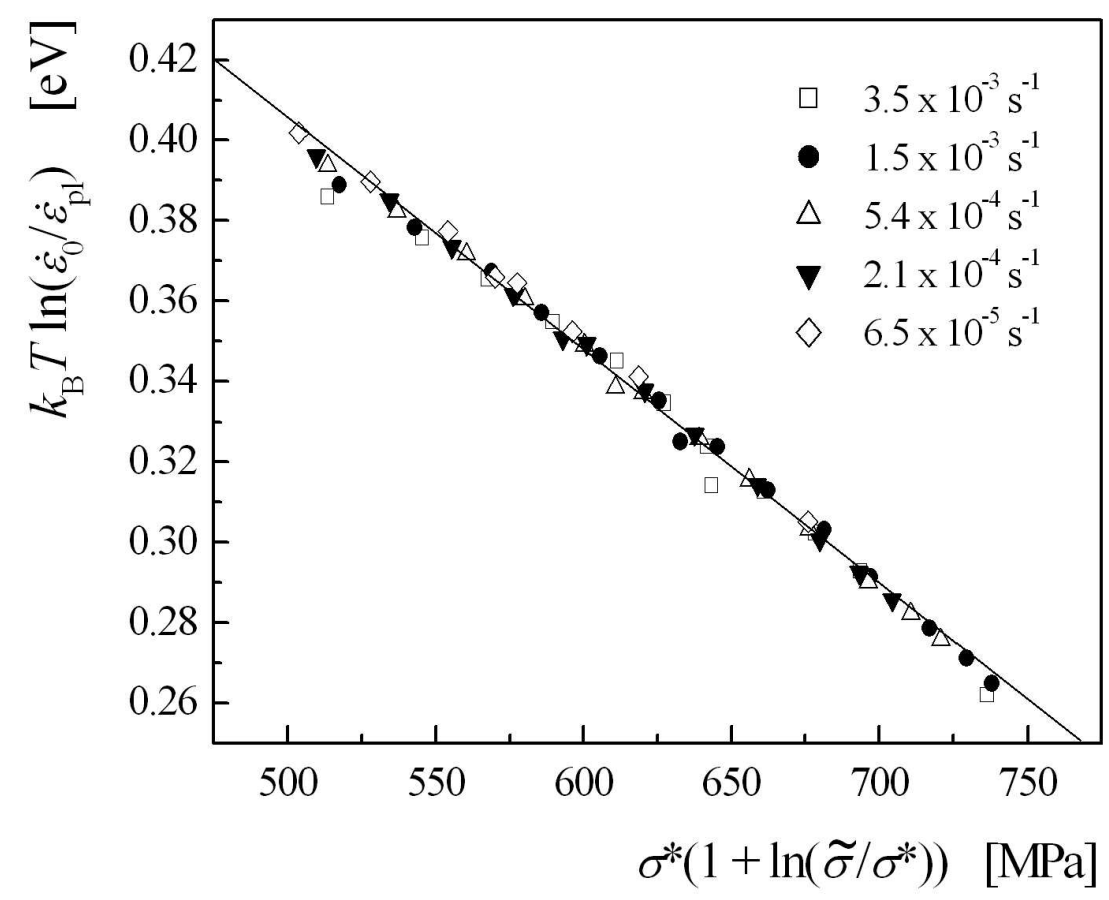

Seeger and Holzwarth Figure 7 $279 \times 215 \mathrm{~mm}(150 \times 150 \mathrm{DPI})$ 


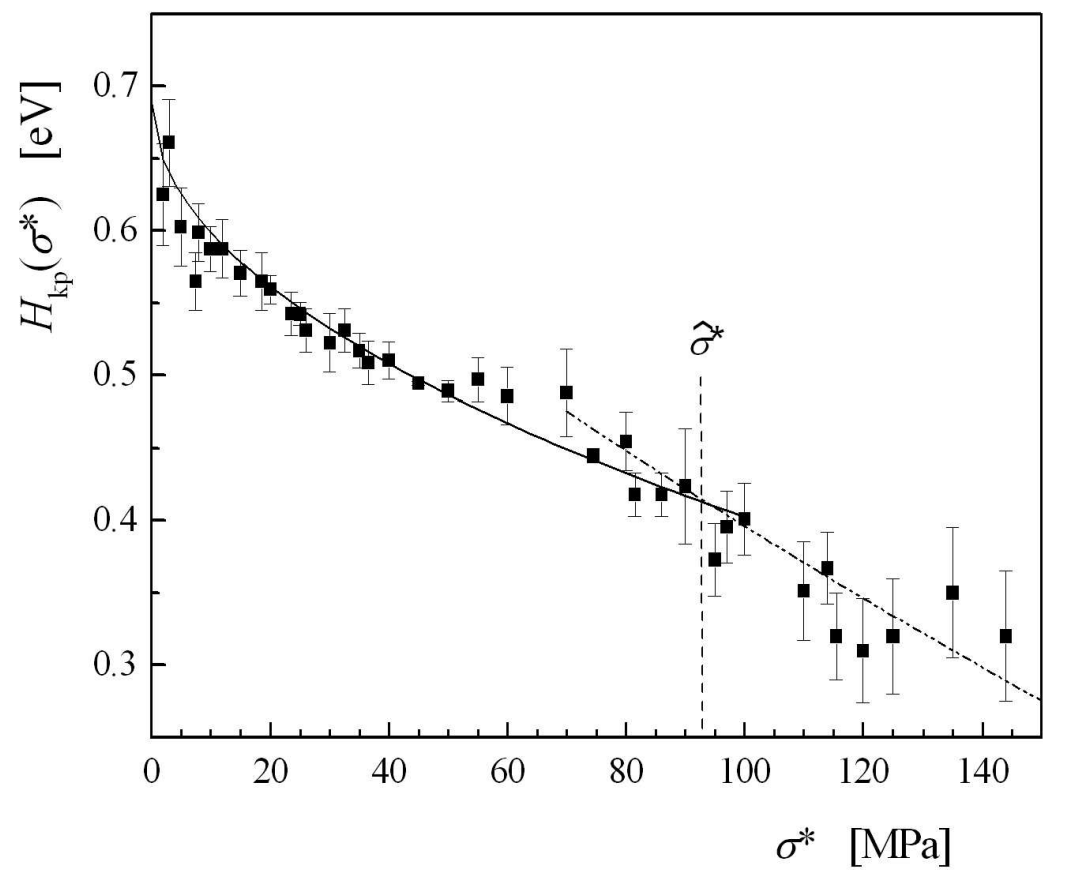

Seeger and Holzwarth Figure 8 $279 \times 215 \mathrm{~mm}(150 \times 150 \mathrm{DPI})$ 


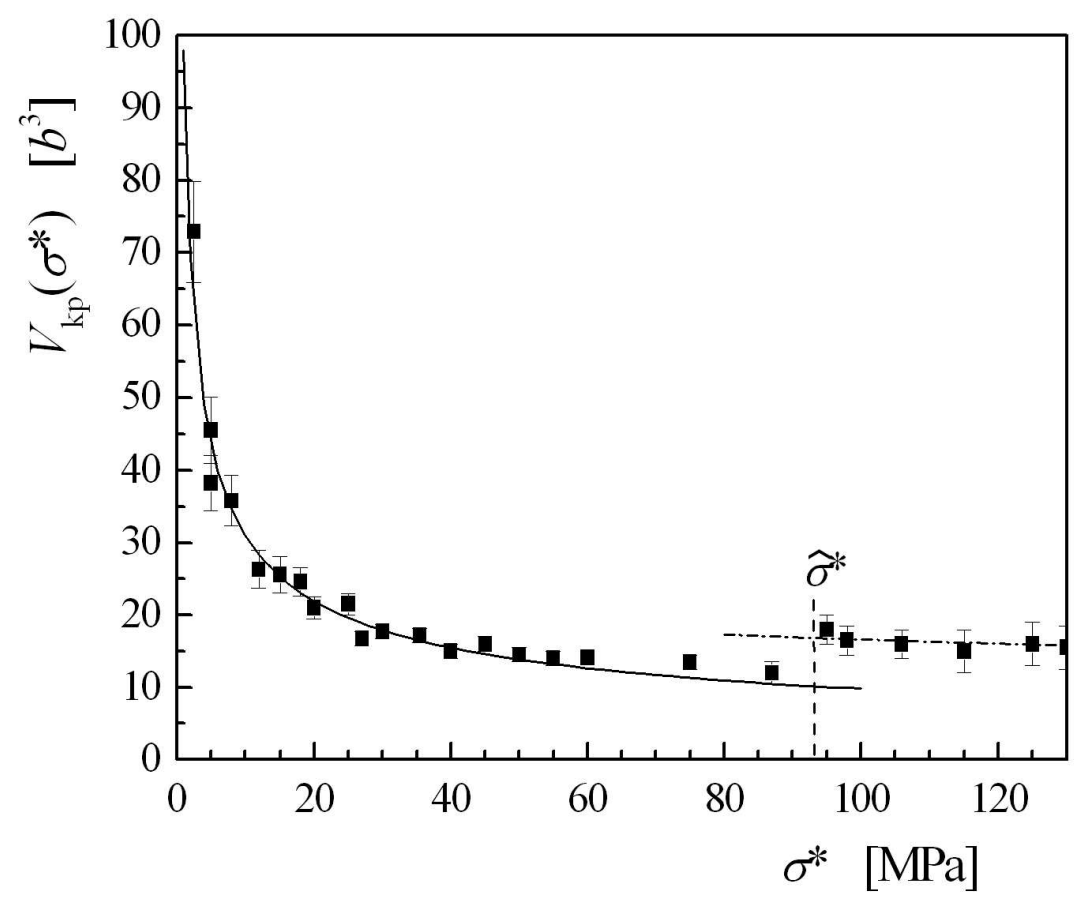

Seeger and Holzwarth Figure 9 $279 \times 215 \mathrm{~mm}(150 \times 150 \mathrm{DPI})$ 


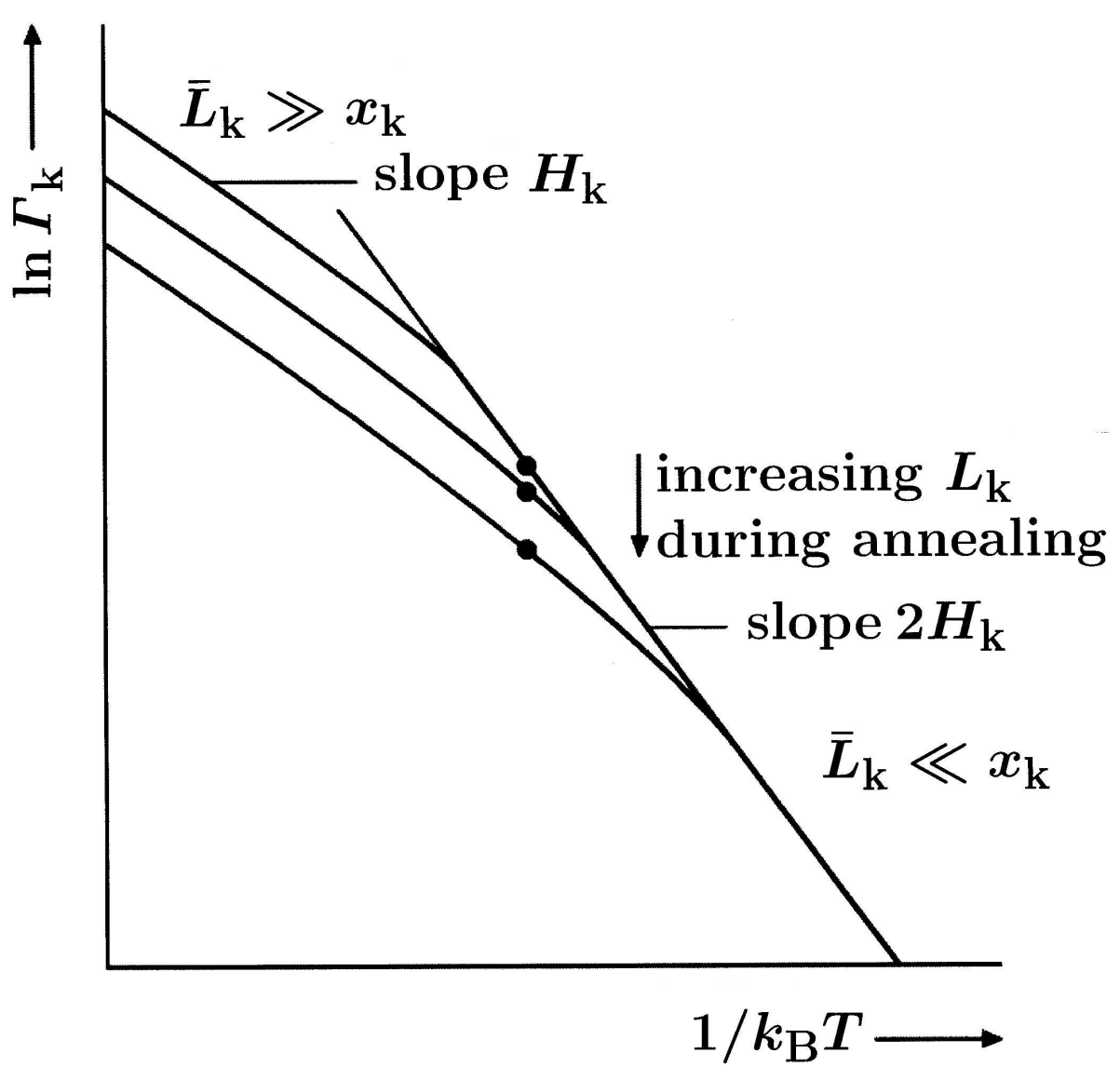

Seeger and Holzwarth Figure 10 $194 \times 195 \mathrm{~mm}(600 \times 600 \mathrm{DPI})$ 


\title{
Slip planes and kink properties of screw dislocations in high-purity niobium
}

\author{
A. SEEGER* and U. HOLZWARTH ${ }^{\dagger}$ \\ Max-Planck-Institut für Metallforschung, Heisenbergstr. 3, D - 70569 Stuttgart, and \\ Universität Stuttgart, Institut für Theoretische und Angewandte Physik, \\ Pfaffenwaldring 57, D - 70569 Stuttgart, Germany
}

Dedicated to Professor Frank Reginald Nunes Nabarro on the occasion of his $90^{\text {th }}$ birthday on March rth, 2006

\footnotetext{
${ }^{*}$ Corresponding Author. Fax: +49-711-685-5271

${ }^{\dagger}$ present address: European Commission, Joint Research Centre, Institute for Health and Consumer Protection, Cyclotron Laboratory, T.P.500, I - 21020 Ispra (VA), Italy
} 
Temperature and strain-rate dependence of the flow stress of cyclically pre-deformed high-purity niobium single crystals have been measured in the temperature range $120 \mathrm{~K} \leq$ $T \leq 350 \mathrm{~K}$ with high accuracy and reproducibility at five or more resolved shear-strain rates between $6.5 \times 10^{-5} \mathrm{~s}^{-1}$ and $3.5 \times 10^{-3} \mathrm{~s}^{-1}$. The data are quantitatively interpreted in terms of the kink-pair generation and kink diffusion in $a_{0}\langle 111\rangle / 2$ screw dislocations $\left(a_{0}=\right.$ cubic lattice parameter). The prediction of a discontinuity in the stress dependence of the activation volume (occasionally dubbed 'the hump') at a strain-rate-independent effective flow stress $\hat{\sigma}^{*}$ has been verified. From the stress dependence of the activation volume and from the magnitude of the discontinuity the spatial period of the Peierls barriers of the screw dislocations could be derived without having to assume a special shape of the Peierls potential. In the temperature range investigated, the measured periodicity is in quantitative agreement with $\{112\}$ as elementary slip planes (i.e., the slip planes of the screw dislocations between cross-slip events) but incompatible with predominant slip on $\{110\}$ planes. Examples of further quantitative results are $\hat{\sigma}^{*}=(93 \pm 5) \mathrm{MPa}$ for the effective stress at the 'upper bend' of the flow-stress-temperature relationship, the zero-stress enthalpy of formation of a pair of isolated kinks, $2 H_{\mathrm{k}}=(0.68 \pm 0.02) \mathrm{eV}$, and the activation energy of kink diffusion, $H_{\mathrm{k}}^{\mathrm{M}}=(15 \pm 10) \mathrm{meV}$. In agreement with the above-mentioned prediction, the same $H_{\mathrm{k}}$ values are obtained above and below $\hat{\sigma}^{*}$. The $\mathrm{Nb}$ data are compared with those on Ta, Mo, W, and $\alpha$-Fe, which all exhibit a similar pattern.

The comparison with the internal-friction measurements of D'Anna and Benoit shows very clearly that the classical $\gamma$-relaxation of $\mathrm{Nb}$ - called irreversible by D'Anna and Benoit is caused by the thermally activated generation of kink pairs in $a_{0}\langle 111\rangle / 2$ screw dislocations on $\{112\}$ planes. For the more recently discovered reversible $\gamma$-relaxation two alternative mechanisms are discussed. The one based on kink-pair formation in screw dislocations on $\{110\}$ planes appears to be the more likely one. This interpretation implies that the reversible $\gamma$-relaxation is identical with the $\beta$-relaxation and thus substantiates Chambers' claim of the intrinsic nature of the $\beta$-relaxation.

keywords: plasticity of metals, dislocation dynamics, refractory metals, mechanical properties, deformation mechanisms, internal friction, Peierls barriers, body-centred cubic metals, kinks in screw dislocations. 


\section{Introduction and General Background}

It is generally accepted that beyond the so-called microstrain deformation regime (sometimes called work-hardening regime 0 [1]) the flow stress of plastically deformed body-centred cubic (bcc) transition metals is controlled by the mobility of screw dislocations with Burgers vectors $\boldsymbol{b}=a_{0}\langle 111\rangle / 2$, where $a_{0}$ denotes the cubic lattice constant. Since, because of the parallelism of Burgers vector and line direction, the slip plane of screw dislocations is not a priory defined, this raises the question of the slip planes of moderately or strongly deformed bcc metals. If slip lines are well developed and straight, by combining them with the slip direction (which may be deduced from the change of the crystal orientation during tensile tests), the slip planes may be determined. In this way it has been found that at low temperatures the $\{110\}$ planes are the dominant slip planes in bulk bcc transition metals.

At higher temperatures the slip lines on the side surfaces of the bcc transition metals are wavy [2]. It is generally accepted that the waviness is a consequence of frequent cross slip of the screw dislocations. Since, however, the length scale on which the screw dislocations change their slip planes by cross slip is unknown, the elementary slip planes, defined as the crystallographic planes on which the screw dislocations slip between two successive cross-slip events, cannot be deduced from wavy slip lines in a straightforward way. This has led to considerable controversies in the literature. Only when the theory of flow-stress control by the generation of kink pairs [3] was applied to the cyclic-deformation technique [4] did it become possible to determine the elementary slip planes in the regime of wavy slip reliably.

The cyclic-deformation technique (also known as Ackermann-Mughrabi technique, to be described in Sect. 3.2) allows us to generate in bcc metals a stable microstructure on which the dependence of the flow stress on both the temperature, $T$, and the resolved plastic strain rate, $\dot{\varepsilon}_{\mathrm{pl}}$, may be measured on one specimen with excellent reproducibility and great accuracy. The determination of the elementary slip planes of screw dislocations by this technique works as follows. The strong temperature and strain-rate dependence of the flow stress of high-purity bcc metals is attributed to Peierls barriers of the screw dislocations that are much larger than those of non-screw dislocations. The screw dislocations overcome their Peierls barriers by stress-assisted generation of pairs of kinks of opposite sign (for short: kink pairs or kinkanti-kink pairs) and subsequent drift of the kinks along the dislocation lines with an average 
velocity $v_{\mathrm{k}}$. In a given crystal structure, the separation of adjacent Peierls barriers, $a_{i j k}$, is uniquely determined by the crystallographic plane $\{i j k\}$ on which the kinks are formed. (Note that kinks in screw dislocations necessarily possess a non-vanishing edge component and, therefore, a well-defined slip plane.) In the bcc structure we have

$$
a_{\{110\}}=(2 / 3)^{1 / 2} a_{0}, \quad a_{\{112\}}=2^{1 / 2} a_{0}=3^{1 / 2} a_{\{110\}}, \quad a_{\{123\}}=(8 / 3)^{1 / 2} a_{0}=2 a_{\{110\}} .
$$

The kink height a, a quantity that can be determined quite accurately by the analysis of the cyclic-deformation data in terms of kink-pair formation (see Sect. 2), is related to $a_{i j k}$ by

$$
a=a_{i j k}
$$

provided the set $\{i j k\}$ of elementary slip planes is unique.

The energy of formation of an isolated kink, $H_{\mathrm{k}}$, and the activation volume of kink-pair formation, $V_{\mathrm{kp}}\left(\sigma^{*}\right)$, are two more quantities that may be deduced with good accuracy from the analysis of the flow-stress data in terms of the kink-pair theory [3]. Following Einstein [5], from the kink formation energy $H_{\mathrm{k}}$ we may derive the kink mass

$$
m_{\mathrm{k}}=H_{\mathrm{k}} / c_{\mathrm{d}}^{2},
$$

where $c_{\mathrm{d}}$ is the speed of the small-amplitude oscillations of the dislocations in their Peierls valleys.

The activation volume $V_{\mathrm{kp}}$ does not have a unique value but depends on the effective resolved shear stress $\sigma^{*}$ acting on the dislocations during the kink-pair generation. The functional dependence $V_{\mathrm{kp}}=V_{\mathrm{kp}}\left(\sigma^{*}\right)$ is highly characteristic of the kink-pair generation process. It permits, therefore, a stringent test of the correctness of the underlying theory, provided that we can relate the effective stress reliably to the measured flow stress $\sigma$.

The determination of $\sigma^{*}=\sigma^{*}\left(T, \dot{\varepsilon}_{\mathrm{pl}}\right)$ starts from the relationship [6]

$$
\sigma=\sigma_{\mathrm{M}}+\sigma^{*}\left(T, \dot{\varepsilon}_{\mathrm{pl}}\right)
$$

Eqn (4) is based on the physical picture that during the plastic deformation preceding the $\sigma^{*}$ measurement a long-range internal stress field had been built up. Evidence for the existence of such a stress field is, inter alia, the Bauschinger effect [7]. The flow stress is controlled by the kink-pair generation at those locations at which the long-range field opposes the dislocation 
motion most strongly. This justifies the linear superposition of the two terms in Eqn (4), where $\pm \sigma_{\mathrm{M}}$ stands for typical extremal values of the internal field. Because of its long-range nature, the $\sigma_{\mathrm{M}}$ contribution cannot be overcome by thermal activation, hence it is strain-rate independent. However, since it originates from the elastic response to the dislocation strain fields, $\sigma_{\mathrm{M}}$ may depend weakly on temperature through the temperature dependence of the elastic moduli, as indicated by the subscript M (see Eqn (4)). As will be discussed in Sect. 4.1 , it is the quite different dependence on $\dot{\varepsilon}_{\mathrm{pl}}$ and $T$ that permits the reliable separation of the two terms of Eqn (4).

The proposal [8] that the kink-pair generation in $a_{0}\langle 111\rangle / 2$ screw dislocations does not only control the flow stress of the refractory bcc metals but is also responsible for the so-called $\gamma$-relaxation in these metals [9] led to the prediction that the two different phenomena should give the same $H_{\mathrm{k}}$ values. This prediction has been verified on several bcc metals including $\mathrm{Nb}[10,11]$. However, more recently the sub-resonance internal-friction measurements of D'Anna and Benoit $[12,13]$ on $\mathrm{Nb}$ and Ta have shown that the $\gamma$-relaxation is a more complex phenomenon than was hitherto thought. The present situation will be discussed in Sects 5 and 6.

The diffusivity $D_{\mathrm{k}}$ of the kinks is a further property that may be deduced by the present approach, though much less accurately so than the kink height $a$, the kink formation energy $H_{\mathrm{k}}$, and the activation volume of kink-pair formation $V_{\mathrm{kp}}\left(\sigma^{*}\right)$. It is related to the kink mobility $\mu_{\mathrm{k}}$ by Einstein's formula

$$
\mu_{\mathrm{k}}=\frac{D_{\mathrm{k}}}{k_{\mathrm{B}} T},
$$

where $k_{\mathrm{B}}$ denotes Boltzmann's constant, and thus determines the speed

$$
v_{\mathrm{k}}=\frac{a b D_{\mathrm{k}} \sigma^{*}}{k_{\mathrm{B}} T}
$$

of kinks drifting along a dislocation line that is acted upon by the effective shear stress $\sigma^{*}$. The knowledge of $D_{\mathrm{k}}$ is important for the quantitative interpretation of the $\alpha^{\prime}$-relaxation, which is attributed to the redistribution of so-called geometrical kinks in screw dislocations that are pinned in different Peierls valleys $[14,15]$.

High-purity niobium was the first material in which cyclic-deformation data were analyzed in terms of the kink-pair generation theory [4]. It turned out that the data below the so-called upper-bend temperature $\hat{T}$ were not sufficient for a critical comparison with the predicted 
$V_{\mathrm{kp}}\left(\sigma^{*}\right)$. The present investigation extends the measurements to lower temperatures and provides more data on the strain-rate dependence, in particular in the temperature regime in which information on the kink diffusivity may be obtained. It uses crystals of similar over-all purity as in the earlier work, as indicated by the residual resistance ratio (see Sect. 3.1). Specific efforts have been made to minimize the possibility of hydrogen contamination (see Sect. 3.3).

The present approach has been stringently tested on ultrapure Mo single crystals, with the result that the agreement between theory and experiment is almost perfect $[16,17]$. It appeared therefore appropriate to start out with an outline of the theory and the underlying assumptions (Sect. 2), to be followed by a fairly detailed description of the experimental procedures (Sect. 3) and the data analysis (Sect. 4). Sect. 5 will treat the relationship between the present work and the $\gamma$-ralaxation.

\section{Theoretical Framework}

In this section we sketch the derivation of the equations relating the kink properties to the experimental data. The contribution of dislocations of strength $b$ and density $N_{\mathrm{d}}$ to the resolved shear-strain rate is

$$
\dot{\varepsilon}_{\mathrm{pl}}=b N_{\mathrm{d}} v_{\mathrm{d}}\left(\sigma^{*}, T\right)
$$

where $v_{\mathrm{d}}$ denotes the stress- and temperature-dependent average speed of the dislocations on their slip plane. Under the conditions of the present experiments $v_{\mathrm{d}}$ is expressed in terms of the rate of kink-pair generation per unit dislocations length, $\Gamma_{\mathrm{k}}=\Gamma_{\mathrm{k}}\left(\sigma^{*}, T\right)$, as

$$
v_{\mathrm{d}}=a \bar{L}_{\mathrm{k}} \Gamma_{\mathrm{k}}\left(\sigma^{*}, T\right)
$$

Eqn (8) is applicable if the drift of the kinks along the dislocation lines is stopped by unsurmountable obstacles with average separation $\bar{L}_{\mathrm{k}}$. If the obstacle separations $L_{\mathrm{k}}$ are very large, the distances travelled by the kinks are limited not by $L_{\mathrm{k}}$ but by the annihilation of kinks of opposite sign. An expression for $v_{\mathrm{d}}$ that allows for both possibilities has been given elsewhere $[15,18]$. In Sect. 6.1 it will be shown that in the present case the simplification leading to Eqn (8) is indeed allowed. 
As described in detail elsewhere [19, 20], the calculation of the kink-pair generation rate $\Gamma_{\mathrm{k}}$ may be reduced to the one-dimensional problem of calculating the escape rate $\Gamma_{1}$ of fictitious particles of mass $m_{\mathrm{k}} / 2$ over a potential barrier. From the partition function of a one-dimensional 'gas' of freely moving kinks it follows that the relationship between $\Gamma_{1}$ and $\Gamma_{\mathrm{k}}$, or, according to Eqns (7) and (8), between $\Gamma_{1}$ and $\dot{\varepsilon}_{\mathrm{pl}}$, contains the temperaturedependent ratio $\Gamma_{\mathrm{k}} / \Gamma_{1} \sim T^{-1 / 2}[19,20]$. It depends on the circumstances and on the quality of the experimental data whether in the temperature dependence of the plastic strain rate $\dot{\varepsilon}_{\mathrm{pl}}$ this factor may be neglected compared with the factor $\exp \left(-H_{\mathrm{kp}} / k_{\mathrm{B}} T\right)$ to be discussed below.

The 'particles' in the one-dimensional problem are assumed to be supplied at the minimum $\Delta E\left(0, \sigma^{*}\right)=0$ of a potential $\Delta E\left(q, \sigma^{*}\right)$ that depends not only on the 'position' $q$ of the 'particles' (equal to the kink-anti-kink separation of a pair of kinks of opposite sign) but also on the effective stress $\sigma^{*}$. Starting from Kramers' theory of thermally activated reactions $[21,22]$, solutions of this problem that are valid in different parameter regimes have been given in the literature $[19,20]$. Since in the original unreduced multidimensional problem the barrier over which the 'particles' have to escape in order for 'free' kinks to be generated is a saddle point of the potential relief, in the one-dimensional formulation we shall continue to refer to the potential barrier, located at $q=q_{\mathrm{s}}$, as the 'saddle point'.

The force causing the 'particles' to drift in the potential $\Delta E\left(q, \sigma^{*}\right)$ is the sum of the contribution $a b \sigma^{*}$ of the effective stress $\sigma^{*}$, and of a contribution deriving from the interaction between the two kinks. At large kink-anti-kink separations $q$, this interaction may be calculated from elasticity theory. The leading term in a series of powers of $1 / q$ is modelindependent and given by $-a^{2} \gamma_{0} / 2 q^{2}$, where $\gamma_{0}$ denotes the pre-logarithmic factor of the dislocation line tension $\gamma_{\mathrm{d}}[23]$. For a given dislocation character and line direction, $\gamma_{0} / b^{2}$ is determined entirely by the second-order elastic constants $c_{i j}$, hence $\gamma_{0}$ may be considered as known. For materials with strong elastic anisotropy such as Nb, the relationship between $\gamma_{0}$ and $c_{i j}$ may be quite complicated, so that numerical computation is required. The recent derivation of an analytical expression for screw dislocations in $\langle 111\rangle$ directions in cubic materials [24] has required considerable effort.

The approach just described is known as elastic-interaction (EI) approximation [3]. Within 
its range of validity, the saddle-point location is determined by

$$
\frac{a^{2} \gamma_{0}}{2 q_{\mathrm{s}}^{2}}=a b \sigma^{*},
$$

hence

$$
q_{\mathrm{s}}=\left(\frac{a \gamma_{0}}{2 b \sigma^{*}}\right)^{1 / 2}
$$

The saddle-point energy is given by

$$
H_{\mathrm{kp}}^{\mathrm{EI}}=2 H_{\mathrm{k}}-\frac{a^{2} \gamma_{0}}{2 q_{\mathrm{s}}}-a b q_{\mathrm{s}} \sigma^{*}=2\left(H_{\mathrm{k}}-\alpha \sigma^{* 1 / 2}\right)
$$

with

$$
\alpha:=\left(\frac{a^{3} b \gamma_{0}}{2}\right)^{1 / 2} .
$$

If considered as a function of the effective stress, Eqn (11) gives us the EI-approximation to the kink-pair formation energy $H_{\mathrm{kp}}\left(\sigma^{*}\right)$. From it follows the activation volume of kink-pair formation as

$$
V_{\mathrm{kp}}=-\left.\frac{\partial H_{\mathrm{kp}}\left(\sigma^{*}\right)}{\partial \sigma^{*}}\right|_{S} \approx-\left.\frac{\partial H_{\mathrm{kp}}\left(\sigma^{*}\right)}{\partial \sigma^{*}}\right|_{T}=\alpha \sigma^{*-1 / 2}=V_{\mathrm{kp}}^{\mathrm{EI}} .
$$

The subscripts $S$ and $T$ indicate that the partial differentiation has to be performed at constant entropy $S$ or, virtually equivalently, at constant temperature $T$.

A further quantity appearing in Kramers' theory is the (negative) curvature of $\Delta E\left(q, \sigma^{*}\right)$ at the saddle point. In terms of the quantity $\lambda_{\mathrm{s}}=\lambda_{\mathrm{s}}\left(\sigma^{*}\right)$ with the dimension of an inverse time defined by

$$
\frac{m_{\mathrm{k}} \lambda_{\mathrm{s}}^{2}}{2}:=\left.\frac{\partial^{2} \Delta E\left(q, \sigma^{*}\right)}{\partial q^{2}}\right|_{\sigma^{*}=\text { const }, q=q_{\mathrm{s}}}
$$

[20], the present approximation gives us

$$
\lambda_{\mathrm{s}}^{2}=\left(b \sigma^{*}\right)^{3 / 2}\left(\frac{2 a}{m_{\mathrm{k}}^{2} \gamma_{0}}\right)^{1 / 2} .
$$

If the kink-anti-kink separation in the saddle point configuration, $q_{\mathrm{s}}$, is less than the critical value

$$
\hat{q}=\left(\frac{a \gamma_{0}}{2 b \hat{\sigma}^{*}}\right)^{1 / 2}
$$

where $\hat{\sigma}^{*}$ will be defined below, the strain fields of the two kinks overlap more than can be handled by the linearized theory of elasticity. Then a physical picture of the kink-anti-kink interaction is required that brings the Peierls potential $U(u)$ to bear. $[U(u)$ is defined as the 
increase of the energy of a straight dislocation line if it is displaced by $u$ from the position of minimum energy.] In this case it is expedient to introduce the so-called line-tension (LT) approximation and to approximate the reaction to changes of the energy stored per unit length in the dislocation strain field by the dislocation line tension

$$
\gamma_{\mathrm{d}}=\gamma_{0} \ln \left(\frac{r_{\infty}}{r_{0}}\right)
$$

In Eqn (17) the inner cut-off radius $r_{0}$ takes into account the failure of the linearized theory of elasticity inside the dislocation core. The outer cut-off radius $r_{\infty}$ is of the order of magnitude of the kink width $w$. A self-consistency procedure gives for $a_{0}\langle 111\rangle / 2$ screw dislocations in $\mathrm{Nb}$ the numerical value $\ln \left(r_{\infty} / r_{0}\right)=2.55[25,26]$.

Since the data of Sect. 4 do not extend to very low temperatures, in their analysis in terms of the LT approximation we may confine ourselves to the leading terms in $\sigma^{*}$. With the abbreviations

$$
\tilde{\sigma}:=2 a U^{\prime \prime}(a) \frac{\exp (\beta)}{b}, \quad U^{\prime \prime}(a):=\left.\frac{\mathrm{d}^{2} U(u)}{\mathrm{d} u^{2}}\right|_{u=a}
$$

and

$$
\begin{aligned}
\beta & :=\int_{u=0}^{a}\left\{u\left[\frac{2 U^{\prime \prime}(a)}{U(u)}\right]^{1 / 2}-\frac{2}{a-u}\right\} \mathrm{d} u \\
& =\int_{\nu=0}^{1}\left\{\nu\left[\frac{2 U^{\prime \prime}(a)}{U(a \nu)}\right]^{1 / 2}-\frac{2}{1-\nu}\right\} \mathrm{d} \nu
\end{aligned}
$$

we then obtain for the activation volume of kink-pair formation

$$
V_{\mathrm{kp}}^{\mathrm{LT}}=a b\left[\frac{\gamma_{\mathrm{d}}}{U^{\prime \prime}(a)}\right]^{1 / 2} \ln \left(\frac{\tilde{\sigma}}{\sigma^{*}}\right)
$$

and for the enthalpy of kink-pair formation

$$
H_{\mathrm{kp}}^{\mathrm{LT}}\left(\sigma^{*}\right)=2 H_{\mathrm{k}}-a b \sigma^{*}\left[\frac{\gamma_{\mathrm{d}}}{U^{\prime \prime}(a)}\right]^{1 / 2}\left[1+\ln \left(\frac{\tilde{\sigma}}{\sigma^{*}}\right)\right] .
$$

The parameter $\beta$ is independent of the strength and the period of the Peierls potential, as may be seen as follows. In the interval $0 \leq u \leq a$ we may write

$$
U(u)=u^{2}(a-u)^{2} f(u / a) \frac{U^{\prime \prime}(a)}{a^{2}},
$$

where $f(u / a)$ is a smooth function satisfying $f(0)=f(1)=0$. Changing the variable of integration to $\eta:=(a-u) / a$ gives us

$$
\beta=2 \int_{\eta=0}^{\eta=1} \eta^{-1}\left[f(\eta)^{-1 / 2}-1\right] \mathrm{d} \eta .
$$


Thus, $\beta$ is indeed entirely determined by the shape function $f(u / a)$. The simplest non-trivial ansatz

$$
f(\eta)=1-\delta \eta(1-\eta)
$$

leads to

$$
\beta=-2 \ln (1-\delta / 4)
$$

The choice $\delta=0$ gives us $\beta=0$. The corresponding Peierls potential $U(u)$, known as Eshelby potential [27], is rather sharply peaked at $u=a / 2$. For potentials that are even more peaked we expect small negative $\beta$. Potentials that near their maxima are flatter than the Eshelby potential lead to positive $\beta$. If we exclude the possibility that the potentials are dented at $u=a / 2$, Eqn (25) gives us $\delta \leq 8 / 3$ and hence $\beta \leq 2 \ln 3 \approx 2.2$. Realistic upper limits of $\beta$ are presumably closer to zero.

The change-over from the EI approximation to the LT approximation does not involve any change in the number or the properties of the dislocations. The strain rate $\dot{\varepsilon}_{\mathrm{pl}}$ is affected only by the change in $H_{\mathrm{kp}}\left(\sigma^{*}\right)$, hence we may define $\hat{\sigma}^{*}$, already introduced in connection with Eqn (16), by

$$
H_{\mathrm{kp}}^{\mathrm{EI}}\left(\hat{\sigma}^{*}\right)=H_{\mathrm{kp}}^{\mathrm{LT}}\left(\hat{\sigma}^{*}\right)
$$

or, taking into account the relationship (17) between $\gamma_{d}$ and $\gamma_{0}$, by

$$
\hat{\sigma}^{*}\left[1+\ln \left(\frac{\tilde{\sigma}}{\hat{\sigma}^{*}}\right)\right]^{2} \ln \left(\frac{r_{\infty}}{r_{0}}\right)=\frac{2 a U^{\prime \prime}(a)}{b} .
$$

A graph representing the solution of Eqn (27) has been given elsewhere [28].

By employing the approximations (13) and (20) up to $\hat{\sigma}^{*}$, we find that $V_{\mathrm{kp}}\left(\sigma^{*}\right)$ should show a sharp bend at $\sigma^{*}=\hat{\sigma}^{*}$. A quantitative measure of the bend is the ratio

$$
\frac{V_{\mathrm{kp}}^{\mathrm{EI}}\left(\hat{\sigma}^{*}\right)}{V_{\mathrm{kp}}^{\mathrm{LT}}\left(\hat{\sigma}^{*}\right)}=\frac{1}{2}\left[1+\frac{1}{\ln \left(\tilde{\sigma} / \hat{\sigma}^{*}\right)}\right] .
$$

It may be obtained experimentally by extrapolating the measured activation volumes $V_{\mathrm{kp}}\left(\sigma^{*}\right)$ towards $\hat{\sigma}^{*}$. From

$$
\ln \left(\frac{\tilde{\sigma}}{\hat{\sigma}^{*}}\right)-2 \ln \left[1+\ln \left(\frac{\tilde{\sigma}}{\hat{\sigma}^{*}}\right)\right]=\beta+\ln \left[\ln \left(\frac{r_{\infty}}{r_{0}}\right)\right]
$$

it follows that $V_{\mathrm{kp}}^{\mathrm{EI}}\left(\hat{\sigma}^{*}\right) / V_{\mathrm{kp}}^{\mathrm{LT}}\left(\hat{\sigma}^{*}\right)$ is determined entirely by dislocation properties and hence independent of the strain rate and, to an excellent approximation, of the measuring temperature. Its measurement allows us to determine the combination $\beta+\ln \left[\ln \left(r_{\infty} / r_{0}\right)\right]$ and, since 
$\ln \left(r_{\infty} / r_{0}\right)$ may be estimated from theory as mentioned above, the shape parameter $\beta$. More on this parameter and its calculation from $U(u)$ may be found in the Appendix.

At moderately high temperatures, at which the effective stress $\sigma^{*}$ is not too low, Kramers' theory [21] gives us for the one-dimensional escape rate referred to above

$$
\Gamma_{1}=\nu_{0} \operatorname{Tgh} \Phi \exp \left\{-\frac{H_{\mathrm{kp}}\left(\sigma^{*}\right)}{k_{\mathrm{B}} T}\right\}
$$

where $\Phi$ is an auxiliary parameter defined by

$$
\operatorname{Sinh} 2 \Phi:=2 m_{\mathrm{k}} \mu_{\mathrm{k}} \lambda_{\mathrm{s}}
$$

$\nu_{0}$ denotes the frequency of the small-amplitude vibrations of straight dislocation lines in their Peierls valleys.

Eqn (30) fails if $\sigma^{*} \rightarrow 0$, as may be seen as follows. The EI-approximation is applicable, hence we may take $\lambda_{\mathrm{s}}$ from Eqn (15) and obtain [20]

$$
\operatorname{Sinh} 2 \Phi:=4 \mu_{\mathrm{k}}\left(b \sigma^{*}\right)^{3 / 4}\left(\frac{2 a m_{\mathrm{k}}^{2}}{\gamma_{0}}\right)^{1 / 4} .
$$

The limit $\sigma^{*} \rightarrow 0$ corresponds to $\Phi \ll 1$. Thus, Eqn (30) reduces, with Eqn (5), to

$$
\Gamma_{1}=2\left(\frac{2 a m_{\mathrm{k}}^{2}}{\gamma_{0}}\right)^{1 / 4} \nu_{0} \frac{D_{\mathrm{k}}}{k_{\mathrm{B}} T}\left(b \sigma^{*}\right)^{3 / 4} \exp \left\{-\frac{H_{\mathrm{kp}}\left(\sigma^{*}\right)}{k_{\mathrm{B}} T}\right\} .
$$

At temperatures $T>\alpha \sigma^{*} / k_{\mathrm{B}}$ one expects, by straightforward physical arguments, $\Gamma_{1} \sim$ $\mu_{\mathrm{k}} \sigma^{*}$. In contrast to this expectation, Eqn (33) predicts $\Gamma_{1} \sim \sigma^{* 3 / 4}$. Thus, as already stated, its validity is restricted to 'moderately' high temperatures. An admittedly somewhat complicated expression that not only gives the correct high-temperature limit but includes Eqn (33) has been derived elsewhere [20].

In the other limit, $\operatorname{Tgh} \Phi \approx 1$, the pre-exponential factor of Eqn $(30)$ is independent of $D_{\mathrm{k}} / k_{\mathrm{B}} T$ and $\sigma^{*}$. This corresponds to the well-known transition-state theory of thermally activated reactions. Thus, in order to deduce the kink-diffusivity $D_{\mathrm{k}}=D_{\mathrm{k}}(T)$ from flow-stress mesurements, we have to go outside the range of validity of the transition state theory, i.e. to fairly small $\sigma^{*}$. A consequence of this is that for the determination of $D_{\mathrm{k}}$ the temperature variation of $\sigma_{\mathrm{M}}$, small as it may be, must be determined as precisely as possible.

The temperature dependence of $D_{\mathrm{k}}$ depends on whether the kink mobility is limited by phonon or electron scattering or by the Peierls barriers of the second kind that kinks 
experience in their motion along dislocation lines. For definiteness, we assume that the latter case obtains and that we may interpret the experimental data in terms of a kink migration enthalpy $H_{\mathrm{k}}^{\mathrm{M}}$ according to

$$
D_{\mathrm{k}}=D_{0} \exp \left\{-\frac{H_{\mathrm{k}}^{\mathrm{M}}}{k_{\mathrm{B}} T}\right\} .
$$

In Sect. 4.2 we shall see that the present experimental results are compatible with this interpretation.

In Fig. 1 in a double-logarithmic plot of $\sigma^{*}$ versus $k_{\mathrm{B}} T / D_{\mathrm{k}}$ (Insert Figure 1 about here.), the uninterrupted full curve marks the division between the two regimes discussed in the two preceding paragraphs. Also shown is the interrupted line $\sigma^{*}=\hat{\sigma}^{*}$ that separates the range of validity of the line-tension approximation from that of the elastic-interaction approximation. Note that these two divisions are independent of each other. Fig. 1 reflects roughly the situation that obtains for the Peierls barriers of $a_{0}\langle 111\rangle / 2$ screw dislocations in refractory bcc metals but may look quite different in other cases. In a properly extended figure two further fields appear in the lower right-hand and the upper left-hand corner, respectively. The first field takes into account that with increasing temperatures more and more kinks are annihilated by anti-kinks before they encounter the 'unsurmountable obstacles' referred to at the beginning of this section, whereas in the second one Kramers' 'classical' theory has to be replaced by a quantum-mechanics treatment that allows for the tunnelling of the 'particles' through the saddle-point barrier. In this regime the knowledge of the kink mass $m_{\mathrm{k}}$ is crucial. The present experiments stay outside both regimes, however.

At $\sigma^{*}<\hat{\sigma}^{*}$, i.e., when the line-tension approximation is valid, the relationship between the effective shear stress and the temperature of deformation may be written as

$$
\sigma^{* 1 / 2}=A\left(T_{\mathrm{K}}-T\right)
$$

with the abbreviation

$$
A:=\frac{k_{\mathrm{B}} \ln \left(\dot{\varepsilon}_{0} / \dot{\varepsilon}_{\mathrm{pl}}\right)}{2 \alpha}
$$

and the so-called knee temperature

$$
T_{\mathrm{K}}=\frac{2 H_{\mathrm{k}}}{k_{\mathrm{B}} \ln \left(\dot{\varepsilon}_{0} / \dot{\varepsilon}_{\mathrm{pl}}\right)} .
$$

The parameter $A$ may be obtained from the slope of $\sigma^{* 1 / 2}$-vs- $T$ plots, the knee temperature $T_{\mathrm{K}}$ by extrapolating these plots towards $\sigma \rightarrow \sigma_{\mathrm{M}}$. Irrespective of whether the pre-exponential 


\section{Experimental Procedures}

\subsection{Material and crystal preparation}

Single crystals oriented for single slip on the plane (101) (Schmid factor $\mu \approx 0.5$ ) were prepared from electrolytically deposited high-purity niobium plates obtained from General Metals Technologies Corporation. According to the manufacturer, the plates contained very low concentrations of substitutional impurities of tantalum, molybdenum and tungsten. They were cut into stripes and remelted by electron-beam melting in high vacuum to bars that were subsequently chemically polished in order to remove impurities taken up during remelting. Cylindrical rods obtained by swaging were purified by electron-beam zone melting, then annealed twice in an oxygen atmosphere of $3 \times 10^{-6}$ mbar to remove dissolved carbon, and at least three times in ultra-high vacuum to remove oxygen, nitrogen, and hydrogen as well as metals with low melting points. The purified rods were swaged to $4 \mathrm{~mm}$ in diameter and chemically polished to $3.8 \mathrm{~mm}$. Single crystals were grown by electron-beam zone melting in ultra-high vacuum by seeding the rods on properly oriented crystals and cut into pieces of $55 \mathrm{~mm}$ length. A well-defined gauge length with a reduced diameter of $3.3 \mathrm{~mm}$ on $10 \mathrm{~mm}$ length was produced by spark erosion. A surface layer of about $0.2 \mathrm{~mm}$ thickness that might possibly have been damaged was removed by chemical polishing in a solution of $15 \mathrm{ml} \mathrm{H}_{2} \mathrm{SO}_{4}$ 
(96\%), $10 \mathrm{ml} \mathrm{HNO}_{3}(65 \%)$, and $12 \mathrm{ml} \mathrm{HF} \mathrm{(40 \% ).} \mathrm{In} \mathrm{order} \mathrm{to} \mathrm{remove} \mathrm{carbon} \mathrm{picked} \mathrm{up}$ during spark erosion, the specimens were once more decarburized in an oxygen atmosphere of $1.5 \times 10^{-6}$ mbar at $2170 \mathrm{~K}$. Hydrogen picked up during polishing was removed by electronbeam heating in ultra-high vacuum for $4 \mathrm{~h}$ at $2570 \mathrm{~K}$.

The residual resistance ratios $(295 \mathrm{~K} / 4.2 \mathrm{~K}$, superconductivity suppressed by a magnetic field) were between $3.5 \times 10^{3}$ and $4 \times 10^{3}$. Under the extreme assumption that a residual resistance ratio of 3500 is due to one kind of impurities only, contamination with 47 atppm hydrogen, 8.5 atppm carbon, 7.3 atppm nitrogen, 8.5 atppm oxygen, or 152 atppm tantalum would account for it $[29,30]$. More realistic is the assumption that the crystals contained all or most of these elements in correspondingly lower concentrations.

\subsection{Pre-deformation procedure and flow stress measurements (Ackermann- Mughrabi technique)}

Prior to measurements involving changes in temperature or strain rate, a well defined stable microstructure was established by cyclic pre-deformation until the maximum flow stress reached during the cycling and the shape of the hysteresis loops did not change any more during further cycling. The resolved shear strain amplitude $\varepsilon_{\mathrm{pl}}=4.8 \times 10^{-3}$ employed in the pre-deformation was large enough to ensure macroyielding by large-scale motion of both non-screw and screw dislocations at the resolved plastic shear-strain rate $\dot{\varepsilon}_{\mathrm{pl}}=1.5 \times 10^{-3} \mathrm{~s}^{-1}$. Establishing stable hysteresis loops required cumulative strains up to $\varepsilon_{\mathrm{pl}, \mathrm{cum}}=4 \times N_{\mathrm{S}} \varepsilon_{\mathrm{pl}}=25$ where $N_{\mathrm{S}}$ denotes the number of cycles after which this 'saturation state' was reached.

The optimal pre-deformation temperature, $T_{\mathrm{pd}}$, was determined by the criteria that the flow stress must be independent of the pre-deformation strain rate $\dot{\varepsilon}_{\mathrm{pl}}$ (this means $T_{\mathrm{pd}}>T_{\mathrm{K}}$ ) and that in the tests at $T<T_{\text {pd }}$ the flow stress must be independent of whether the temperature $T$ was reached from above or below. The latter criterion guarantees the statistical stability of the microstructure during the tests. It was fulfilled for $T_{\mathrm{pd}} \geq 350 \mathrm{~K}$. In order to stay on the safe side, $T_{\mathrm{pd}}=370 \mathrm{~K}$ was chosen for the measurements to be reported in Sect. 4. In agreement with the electron-microscopy observations of Luft et al. [31] on molybdenum single crystals, deformation cycles above the pre-deformation temperature provoked rearrangements of the dislocation pattern. 
After pre-deformation, the temperature was lowered in steps of $5 \mathrm{~K}$. At each temperature, hysteresis loops were recorded at plastic strain rates $\dot{\varepsilon}_{\mathrm{pl}}$ ranging from $6.5 \times 10^{-5} \mathrm{~s}^{-1}$ to $3.5 \times 10^{-3} \mathrm{~s}^{-1}$. After about 50 cycles at different temperatures and strain rates, the specimens were cycled for some ten cycles at the above-mentioned pre-deformation conditions in order to eliminate small changes in the microstructure that might have occurred. The initial microstructure was considered restored when the saturation flow stress of the pre-deformation measurements had been reached again. By repeating this procedure, several hundreds of flow-stress measurements at different temperatures and strain rates could be performed on one single specimen at approximately constant microstructure and constant crystallographic orientation of the load axis.

Constant plastic strain rates $\dot{\varepsilon}_{\mathrm{pl}}$ were achieved in a servo-hydraulic closed-loop-controlled MTS system using a triangular command signal for plastic strain. Axial strain of the specimen and load were measured with an extensometer and a load cell, respectively. A dual-slope function generator permitted changes of $\dot{\varepsilon}_{\mathrm{pl}}$ during a deformation cycle when a given percentage of the plastic strain amplitude $\varepsilon_{\mathrm{pl}}$ was reached. In the present experiments this percentage was set to $75 \%$. (For a more complete description of the method, which allowed us to minimize changes of microstructure when changing to higher $\dot{\varepsilon}_{\mathrm{pl}}$, to reduce time consumption and to minimize stress relaxation effects during deformation at very small $\dot{\varepsilon}_{\mathrm{pl}}$, see [16].) From the absolute values of the peak loads of the hysteresis loops $F_{\mathrm{t}}$ in tension and $F_{\mathrm{C}}$ in compression the resolved flow stress was calculated according to

$$
\sigma=\mu \frac{\left|F_{\mathrm{t}}\right|+\left|F_{\mathrm{c}}\right|}{A}
$$

where $A$ denotes the cross sectional area of the gauge length of the crystals and $\mu$ the Schmid factor for $[111](\overline{1} 01)$ slip.

The specimens were deformed at $120 \mathrm{~K} \leq T \leq 295 \mathrm{~K}$ in an isopentane bath that was cooled by liquid nitrogen flowing through a coil controlled by a solenoid valve, and at $295 \mathrm{~K} \leq T \leq$ $370 \mathrm{~K}$ in a silicon oil bath that was heated with a nickel-chromium conductor connected to a controllable DC power supply. In both cases the constancy of temperature was better than $\pm 0.2 \mathrm{~K}$. 


\subsection{Avoidance of hydrogen contamination}

Considerable effort was spent on avoiding contamination with hydrogen during plastic deformation. In preliminary experiments it was found that after the pre-deformation at $370 \mathrm{~K}$ the residual resistance had increased by much more than could be accounted for by the increase of the dislocation density. This was attributed to contamination by hydrogen. Internal friction measurements indicated indeed that at the end of the deformation experiments the hydrogen content exceeded the starting concentration by orders of magnitude, which could have been at most 50 atppm (cf. Sect 3.1) but was presumably distinctly lower. A thin oxide layer did not prevent the hydrogen contamination because during deformation it was continuously destroyed by the formation of slip steps.

The contamination problem was solved by fitting the crystals into small elastic metallic vacuum chambers that were deformed together with the samples, thus avoiding direct contact with the temperature bath. As shown in the longitudinal cut of Fig. 2 (Insert Figure 2 about here.), the chambers consisted of two parallel discs with cylindrical extensions, housing the gripping parts of the crystals, and of a metal-bellow segment enclosing the space between the discs that housed the gauge length of the crystals. The metal-bellows segment allowed easy elastic deformation of the vacuum chamber. The discs and their cylindrical extensions were machined from a single piece of steel to ensure vacuum tightness. The fabrication started by drilling a hole along the axis of a round steel rod of $40 \mathrm{~mm}$ in diameter, which exactly matched the crystal diameter on the gripping part. The material around this hole was removed by machining until only a thin wall of $0.2 \mathrm{~mm}$ thickness was left. When mounting the specimens on the fatigue machine this thin-walled extensions of the vacuum chambers were deformed in a way that ensured sufficient gripping contact between the specimens and the machine fixtures as well as vacuum tightness. A small tube attached to the upper lid permitted evacuating the chamber. The upper and lower component of the vacuum chamber were assembled with the metal-bellow element and the specimen in between them. All connections between the discs and the metal bellows were made with a special vacuum adhesive (ECCOBOND, Emerson \& Cuming Inc.). Slitted iron spacers were used to adjust the diameter of the chamber on the extensions to the grips of the MTS-machine. The force needed to deform the chamber was negligible compared with that required to deform 


\section{Experimental Results and Analysis}

\subsection{Determination of $\sigma^{*}\left(T, \dot{\varepsilon}_{\mathrm{pl}}\right)$ and $\hat{\sigma}^{*}$}

At and above $T=350 \mathrm{~K}$, changing $\dot{\varepsilon}_{\mathrm{pl}}$ did not affect the flow stress $\sigma$, hence the saturation flow stress reached during pre-deformation cycling was identified with $\sigma_{\mathrm{M}}=\sigma-\sigma^{*}$. On the single crystal $\mathrm{Nb} 95 / 1$, which was deformed in vacuum as described in Sect. 3.3 and on which the data to be presented below were obtained unless otherwise stated, we found $\sigma_{\mathrm{M}}(370 \mathrm{~K})=17.5 \mathrm{MPa}$. Although independent of $\dot{\varepsilon}_{\mathrm{pl}}$ (cf. Sect. 3.2), $\sigma_{\mathrm{M}}(350 \mathrm{~K})$ was slightly but clearly measurably larger than $\sigma_{\mathrm{M}}(370 \mathrm{~K})$. This indicated the need for the correction for the temperature dependence of the elastic moduli alluded in Sects 1 and 2. We thus replaced Eqn (4) by

$$
\sigma^{*}\left(T, \dot{\varepsilon}_{\mathrm{pl}}\right)=\sigma\left(T, \dot{\varepsilon}_{\mathrm{pl}}\right)-\sigma_{\mathrm{M}}\left(T_{\mathrm{p}}\right) \frac{M_{\mathrm{sh}}(T)}{M_{\mathrm{sh}}\left(T_{\mathrm{p}}\right)} .
$$

The shear modulus $M_{\text {sh }}$ for screw dislocations in $\langle 111\rangle$ directions was calculated according to Chou and Sha [32] using the temperature dependence of the elastic compliancies $s_{i j}$ of Armstrong et al. [33]. This gave an increase of $\sigma_{\mathrm{M}}$ between $370 \mathrm{~K}$ and $170 \mathrm{~K}$ by about $5 \%$.

Fig. 3 (Insert Figure 3 about here.) shows the results of a few hundred measurements at five different strain rates. The regularity of the data demonstrates that the microstructure 
established by the cyclic pre-deformation at $370 \mathrm{~K}$ was indeed stable in a statistical sense. The $T$-dependence of the flow stress shows a strain-rate-independent bend at $\sigma^{*}=(93 \pm 5) \mathrm{MPa}$, which we therefore identify with the stress $\hat{\sigma}^{*}$ introduced by Eqn (26). The strain-ratedepending upper-bend temperatures $\hat{T}$ are defined by $\sigma^{*}\left(\hat{T}, \dot{\varepsilon}_{\mathrm{pl}}\right):=\hat{\sigma}^{*}$.

\subsection{The regime of low effective stresses $\left(\sigma^{*}<\hat{\sigma}^{*}\right)$}

The 'upper bend' of $\sigma^{*}\left(T, \dot{\varepsilon}_{\mathrm{pl}}\right)$ at $\hat{\sigma}^{*}=(93 \pm 5) \mathrm{MPa}$ is brought out very clearly in Fig. 4 (Insert Figure 4 about here.), where the $\sigma^{*}-T$ data have been plotted according to Eqn (35). The deviations from straight lines on the high-temperature side of the plot are due to the failure of the transition-state theory and contain, according to Sect. 2, information on the kink diffusivity. The boundary between the applicability of the approximations Eqn (33) and the transition-state approximation

$$
\Gamma_{1}=\nu_{0} \exp \left\{-\frac{H_{\mathrm{kp}}\left(\sigma^{*}\right)}{k_{\mathrm{B}} T}\right\}
$$

is marked by the dash-dotted line (see Figs 3 and 4). The increase of $-\partial \sigma^{*} /\left.\partial T\right|_{\dot{\varepsilon}_{\mathrm{pl}}}$ with decreasing temperature that sets in at the so-called 'lower bend' temperature $\check{T}$ occurs at too low temperatures to be investigated with the present cooling technique. It is attributed to a transformation of the screw-dislocation cores to a configuration that allows them to slip on $\{110\}$ planes [34].

In analyzing the measurements in the regime $\sigma^{*}<\hat{\sigma}^{*}$ quantitatively, we have the choice between a 'global' fit of the data based on Eqn (30) with unrestricted $\Phi$, or separate fits to Eqn (33), derived for the regime controlled by kink diffusion, or Eqn (42), following from by the transition-state approximation. Global fits were performed for similar but considerably larger data sets on molybdenum single crystals by Hollang et al. [16]. We have developed a different procedure that starts out with the determination of the knee temperature $T_{\mathrm{K}}$ by means of Eqn (35) and connects later (Sect. 4.3) the EI results with the LT approximation. The 'knee temperatures' $T_{\mathrm{K}}$ obtained by extrapolating the fits to Eqn (35) to $\sigma^{*}=0$ are listed in Fig. 4. As Fig. 5 shows (Insert Figure 5 about here.), they obey very well the linear relationship (37) between $1 / T_{\mathrm{K}}$ and $\ln \dot{\varepsilon}_{\mathrm{pl}}$. We obtain $\dot{\varepsilon}_{0}=1.14 \times 10^{8} \mathrm{~s}^{-1}$ from the ordinate intersection and $2 H_{\mathrm{k}}=(0.68 \pm 0.01) \mathrm{eV}$ from the slope. Eqn (12) then gives us $\alpha:=\left(a^{3} b \gamma_{0} / 2\right)^{1 / 2}=(2.27 \pm 0.05) \times 10^{-27} \mathrm{~m}^{2} \mathrm{~N}^{1 / 2}$. As argued in Sect. 1 , we attribute 
these values to screw dislocations with Burgers vector $\boldsymbol{b}=a_{0}\langle 111\rangle / 2$. With $b=3^{1 / 2} a_{0} / 2$, $a_{0}=3.3007 \times 10^{-10} \mathrm{~m}$, and $\gamma_{0}=3.4 \times 10^{-10} \mathrm{~N}[4,35]$, we obtain the kink height

$$
a=(1.43 \pm 0.05) a_{0}=(4.73 \pm 0.15) \times 10^{-10} \mathrm{~m} .
$$

Eqn (43) is in almost perfect agreement with $a_{\{112\}}$ (cf. Eqns (1)). Its incompatibility with $a_{\{110\}}$ demonstrates that $\{110\}$ planes are definitely not the dominant slip planes of highpurity niobium single crystals in the temperature range $180 \mathrm{~K} \leq T \leq 250 \mathrm{~K}$. Good agreement of the measured $a$ with $a_{\{112\}}$ was found also in other refractory bcc metals investigated (Ta $[36,37]$, Mo [16], W [38], $\alpha$-Fe [39]; see Table 1). Since it is extremely unlikely that mixtures of different kink height, e.g. of $a_{\{110\}}$ and $a_{\{123\}}$, always give the same result, the conclusion that $\{112\}$ was the dominant slip plane in the present experiments is compelling.

As already emphasized, above about $220 \mathrm{~K}$ we may obtain information on the kinkdiffusivity $D_{\mathrm{k}}$. The $D_{\mathrm{k}}$ values shown in Fig. 6 (Insert Figure 6 about here.) as open squares were deduced from the measurements above $220 \mathrm{~K}$ at $\dot{\varepsilon}_{\mathrm{pl}}=1.5 \times 10^{-3} \mathrm{~s}^{-1}$ by combining Eqns (7), (8), (21) and (33), making use of the $H_{\mathrm{k}}, \alpha$ and $\dot{\varepsilon}_{0}$ values derived by the preceding fitting procedure. The tendency of these preliminary values to decrease with increasing temperature suggested the description of the kink diffusivity by the Arrhenius law (34). For extra accuracy, flow stress measurements were performed at 10 different strain rates at $220 \mathrm{~K}, 240 \mathrm{~K}, 260 \mathrm{~K}, 280 \mathrm{~K}, 300 \mathrm{~K}, 320 \mathrm{~K}$ and $340 \mathrm{~K}$. Using preliminary $H_{\mathrm{k}}^{\mathrm{M}}$ and $D_{0}$ obtained in this way as additional starting values, we carried out a 'pseudo-global' least-square fit of Eqns (7), (8), (21) and (33) to the flow-stress data $\sigma^{*}<\hat{\sigma}^{*}$ with the restriction that $H_{\mathrm{k}}, \alpha$ and $H_{\mathrm{k}}^{\mathrm{M}}$ should stay within the uncertainties of the previous fits. The $D_{\mathrm{k}}$ values found in this way are shown in Fig. 6 as full circles and may be described by

$$
H_{\mathrm{k}}^{\mathrm{M}}=(15 \pm 10) \mathrm{meV}, \quad D_{0}=(0.9 \pm 0.3) \times 10^{-6} \mathrm{~m}^{2} \mathrm{~s}^{-1}
$$

The temperature range over which $D_{\mathrm{k}}$ could be deduced is too narrow to establish with certainty that the kink diffusivity does obey Eqn (34). Support for this interpretation comes from the order of magnitude of the pre-exponential factor $D_{0}$ according to the following argument. The period of the Peierls potential of the second kind experienced by the kinks migrating along $a_{0}\langle 111\rangle / 2$ screw dislocations is $b=2.858 \times 10^{-10} \mathrm{~m}$. The attempt frequency $\nu_{\mathrm{k}}^{0}=D_{0} / b^{2} \approx 10^{-13} \mathrm{~s}^{-1}$ following from the transition-state theory in its simplest form (en- 
tropy terms neglected) is of the order of magnitude of the frequency of short-wavelength lattice vibrations, as expected.

\subsection{The regime of intermediate effective stresses $\left(\sigma^{*}>\hat{\sigma}^{*}\right)$}

Making use of the strain-rate dependent $T_{\mathrm{K}}$ values of Sect. 4.2, a fit of the effective stresses $\sigma^{*}>\hat{\sigma}^{*}$ to Eqn (39) yields $2 H_{\mathrm{k}}=(0.69 \pm 0.02) \mathrm{eV}, \tilde{\sigma}=(7.1 \pm 0.2) \mathrm{GPa}$ and $a b\left[\gamma_{\mathrm{d}} / U^{\prime \prime}(a)\right]^{1 / 2}=$ $(9.1 \pm 0.2) \times 10^{-29} \mathrm{~m}^{3}$. These fit values may be cross-checked by rewriting Eqn (39) as

$$
k_{\mathrm{B}} T \ln \left(\frac{\dot{\varepsilon}_{0}}{\dot{\varepsilon}_{\mathrm{pl}}}\right)=2 H_{\mathrm{k}}-\left[a b\left(\frac{\gamma_{\mathrm{d}}}{U^{\prime \prime}(a)}\right)^{1 / 2}\right] \sigma^{*}\left[1+\ln \left(\frac{\tilde{\sigma}}{\sigma^{*}}\right)\right]
$$

and plotting $k_{\mathrm{B}} T \ln \left(\dot{\varepsilon}_{0} / \dot{\varepsilon}_{\mathrm{pl}}\right)$ versus $\sigma^{*}\left[1+\ln \left(\tilde{\sigma} / \sigma^{*}\right)\right]$ (see Fig. 7). (Insert Figure 7 about here.). From the slope in Fig. 7 we get

$$
\left[a b\left(\frac{\gamma_{\mathrm{d}}}{U^{\prime \prime}(a)}\right)^{1 / 2}\right]=(9.13 \pm 0.13) \times 10^{-29} \mathrm{~m}^{3} .
$$

From the ordinate intersection we obtain $2 H_{\mathrm{k}}=0.68_{5} \pm 0.01_{5} \mathrm{eV}$, which agrees with the result of the data analysis in the EI regime. The uncertainty of $\tilde{\sigma}$ was without significant effect on the quantities deduced from Fig. 7.

Eqn (46) allows us to derive the kink height $a$ making use of the data obtained at $\sigma^{*}>\hat{\sigma}^{*}$ only. We eliminate $\ln \left(r_{\infty} / r_{0}\right)$ from Eqn (27) with the help of Eqn (17), solve for $\gamma_{\mathrm{d}} / U^{\prime \prime}(a)$, and insert the result into Eqn (46). With the numerical values $\hat{\sigma}^{*}=93 \mathrm{MPa}, \tilde{\sigma}=7.1 \mathrm{GPa}$, $\gamma_{0}=3.4 \times 10^{-10} \mathrm{~N}$ and $b=2.858 \times 10^{-10} \mathrm{~m}$, we then find $a=4.84 \times 10^{-10} \mathrm{~m}$ in agreement with the result Eqn (43) obtained at $\sigma^{*}<\hat{\sigma}^{*}$.

Thus, kink energy $2 H_{\mathrm{k}}$ and kink height $a$ come out the same irrespective of whether they are deduced in the EI regime or in the LT regime. One could hardly wish a more convincing demonstration that the flow stress is determined by the same mechanism on both sides of the 'hump' at $\hat{\sigma}^{*}$.

Finally we obtain from Eqn $(27) U^{\prime \prime}(a)=(2.0 \pm 0.1)$ GPa and from Eqn $(18) \beta=0.06 \pm$ 0.05 .

\subsection{The stress dependence $H_{\mathrm{kp}}\left(\sigma^{*}\right)$ and $V_{\mathrm{kp}}\left(\sigma^{*}\right)$}

The stress dependence of the kink-pair formation enthalpy $H_{\mathrm{kp}}\left(\sigma^{*}\right)$ and the activation volume of kink-pair formation $V_{\mathrm{kp}}\left(\sigma^{*}\right)$ may be derived with a minimum of assumptions from the 
strain-rate sensitivity of the flow stress at constant temperature, $\partial \sigma^{*} /\left.\partial \ln \dot{\varepsilon}_{\mathrm{pl}}\right|_{T}$, and the temperature dependence of the effective flow stress at constant plastic strain-rate, $\partial \sigma^{*} /\left.\partial T\right|_{\dot{\varepsilon}_{\mathrm{pl}}}$ [40]. Both quantities may be obtained by the present experimental techniques.

The assumptions are:

(1) Temperature and strain-rate dependence of the plastic strain rate $\dot{\varepsilon}_{\mathrm{pl}}$ are essentially determined by the Arrhenius factor $\exp \left\{-H_{\mathrm{kp}}\left(\sigma^{*}\right) / k_{\mathrm{B}} T\right\}$, where the activation enthalpy $H_{\mathrm{kp}}\left(\sigma^{*}\right)$ does not explicitly depend on temperature.

(2) Any temperature or stress dependence due to the pre-exponential factor is small compared with that due the Arrhenius factor and at least approximately known.

If the stress dependence of the pre-exponential factor is negligible, the activation volume is given by the well-known expression

$$
V_{\mathrm{kp}}\left(\sigma^{*}\right)=\left.k_{\mathrm{B}} T \frac{\partial \ln \dot{\varepsilon}_{\mathrm{pl}}}{\partial \sigma^{*}}\right|_{T}
$$

In contrast to $V_{\mathrm{kp}}\left(\sigma^{*}\right)$, the expression for $H_{\mathrm{kp}}\left(\sigma^{*}\right)$ is influenced by the $T$-dependence of the pre-exponential factor. In the special case that the pre-exponential is proportional to $T^{-1 / 2}$, the activation enthalpy may be obtained from

$$
H_{\mathrm{kp}}\left(\sigma^{*}\right)=\frac{1}{2} k_{\mathrm{B}} T-\left.k_{\mathrm{B}} T^{2} \frac{\partial \sigma^{*}}{\partial T}\right|_{\dot{\varepsilon}_{\mathrm{pl}}}\left\langle\left.\frac{\partial \sigma^{*}}{\partial \ln \dot{\varepsilon}_{\mathrm{pl}}}\right|_{T} .\right.
$$

Fig. 8 compares the $H_{\mathrm{kp}}\left(\sigma^{*}\right)$ values obtained from Eqn $(48)$ with $H_{\mathrm{kp}}^{\mathrm{EI}}\left(\sigma^{*}\right)$ and $H_{\mathrm{kp}}^{\mathrm{LT}}\left(\sigma^{*}\right)$ as calculated from Eqns (11) and (21) with $2 H_{\mathrm{k}}=0.69 \mathrm{eV}$ and $a=4.8 \times 10^{-10} \mathrm{~m}, U^{\prime \prime}(a)=$ $2.0 \mathrm{GPa}, \tilde{\sigma}=7.1 \mathrm{GPa}$, and $\ln \left(r_{\infty} / r_{0}\right)=2.55$. The agreement is good, in particular in the stress regime where experimental errors are small. The $\sigma^{* 1 / 2}$-rise of $H_{\mathrm{kp}}\left(\sigma^{*}\right)$ at $\sigma^{*} \rightarrow 0$ and the break in the slope at $\hat{\sigma}^{*}$ is clearly visible.

The comparison of $V_{\mathrm{kp}}\left(\sigma^{*}\right)$ as obtained from Eqn (47) with the curves computed from Eqns (13) and (20) in Fig. 9 shows the predicted discontinuity at $\hat{\sigma}^{*}=93 \mathrm{MPa}$ even more clearly. Also clearly visible is the $\sigma^{*-1 / 2}$ divergence of $V_{\mathrm{kp}}\left(\sigma^{*}\right)$ towards $\sigma^{*}=0$. It may be used to test whether $\sigma_{\mathrm{M}}$ has been chosen correctly. Activation volumes between $20 b^{3}$ and $50 b^{3}$ as they have been observed at small $\sigma^{*}$ are very typical for the stress-assisted kink-pair generation in the refractory bcc metals, independent of the Peierls potential $U(u)$. 


\section{Comparison flow stress $-\gamma$-relaxation}

In the early 1960s, Chambers [9] introduced a nomenclature of the relaxation processes in the refractory bcc metals that has remained in use till now with small modifications, such as the distinction between the $\alpha$-relaxation (attributed to kink-pair generation in non-screw dislocations) and the $\alpha$ '-relaxation (attributed to the migration of geometrical kinks in $a_{0}\langle 111\rangle / 2$ screw dislocations). In 1971, it was proposed that the $\gamma$-relaxation is due to the stress-assisted generation of kink pairs in screw dislocations, i.e to the flow-stress-controlling process discussed in the present paper [8]. The attribution of the $\gamma$-relaxation and of the strain-rate and temperature dependence of the flow stress to the same process is not trivial and should therefore be subjected to experimental tests, since the Ackermann-Mughrabi technique investigates the flow stress on dislocation densities far beyond those of the micro-strain regime, whereas relaxation studies by means of low-amplitude internal friction measurements are usually performed on much lower dislocation densities at or close to micro-strain conditions.

The physical quantity most suitable for a quantitative comparison is the enthalpy of formation of a pair of widely separated kinks, $2 H_{\mathrm{k}}$. In the flow-stress measurements it is obtained as the limit $\sigma^{*} \rightarrow 0$ of $H_{\mathrm{kp}}\left(\sigma^{*}\right)$, in the internal friction measurements by analyzing the shift of the temperature of internal friction peaks as a function of the measuring frequency $f$. The agreement of the two types of measurements found in many cases (for recent reviews see $[11,15])$ allowed us to conclude that the $\gamma$-relaxation involves the generation of kink pairs on the $\{112\}$ slip planes of screw dislocations [10]. This conclusion has opened the door for the understanding of the $\beta$-relaxation of the Chambers nomenclature, which for decades had remained without a convincing explanation. The $\beta$-relaxation appears now to be due to the kink-pair generation of $a_{0}\langle 111\rangle / 2$ screw dislocations, too, though not on $\{112\}$ planes but on $\{110\}$ slip planes [41]. This assignment, derived with the help of the flow-stress measurements, has important consequences for the following.

An entirely unexpected result was revealed by the internal friction work of D'Anna and Benoit [12] on slightly deformed $\mathrm{Nb}$ and Ta. In extensive measurements based on isothermal frequency sweeps in the range $10^{-4} \mathrm{~Hz} \leq f \leq 10^{-1} \mathrm{~Hz}$, these authors made the surprising discovery that in the temperature range of the $\gamma$-relaxation two relaxation peaks may appear that respond quite differently to moderate anneals. In $\mathrm{Nb}$, after a deformation of about 
$2 \times 10^{-3}$ at about $190 \mathrm{~K}$, the fit of the relaxation times

$$
\tau=\tau_{\infty} \exp \left\{\frac{H_{\gamma}}{k_{\mathrm{B}} T}\right\}
$$

determined in a first run at increasing temperatures up to $280 \mathrm{~K}$ gave

$$
H_{\gamma}=(0.68 \pm 0.03) \mathrm{eV}, \tau_{\infty}=1.2 \times 10^{-13} \mathrm{~s}^{-1}
$$

in good agreement with $2 H_{\mathrm{k}}^{\{112\}}$ as derived from the present flow-stress measurements. After annealing at $290 \mathrm{~K}$, an internal-friction peak was still observed in roughly the same temperature regime as before but now with

$$
H_{\gamma}=(0.59 \pm 0.03) \mathrm{eV}, \tau_{\infty}=7 \times 10^{-12} \mathrm{~s}^{-1}
$$

This peak proved stable against annealing at $290 \mathrm{~K}$, in contrast to the peak described by Eqn (50). Because of the different annealing behaviour D'Anna and Benoit denoted the peak with the lower activation enthalpy as reversible $\gamma$-peak and the peak with the higher activation enthalpy as irreversible $\gamma$-peak.

A most surprising and difficult-to-understand aspect of the preceding results is that it is the unstable peak that is fully compatible with the results of the flow-stress measurements, whereas the activation energy of the stable peak is clearly incompatible with $2 H_{\mathrm{k}}^{\{112\}}$. In the following we consider two interpretation proposals that start from opposite assumptions and that, therefore, should not be too difficult to distinguish by appropriately designed experiments. Common to both proposals and in agreement with the now accepted view [8] is that both peaks are attributed to the same dislocations, namely $a_{0}\langle 111\rangle / 2$ screw dislocations.

Proposal (a) assumes that the annealing removes obstacles to the kink motion along the screw dislocations (e.g. jogs, dislocation nodes) to such an extent that the condition $\bar{L}_{\mathrm{k}} \ll x_{\mathrm{k}}$, where $x_{\mathrm{k}}$ denotes the mean distance of the kinks in thermal equilibrium, is violated (cf. Sect. 2). The consequences of this for the temperature dependence of the net rate of kink-pair generation, $\Gamma_{\mathrm{k}}$, is illustrated in Fig. 10 [18, 42]. (Insert Figure 10 about here.) Whereas at $\bar{L}_{\mathrm{k}} \ll x_{\mathrm{k}}$, the case so far considered exclusively, the activation energy is given by $2 H_{\mathrm{k}}$, it decreases gradually towards $H_{\mathrm{k}}$ as $\bar{L}_{\mathrm{k}}$ increases. (For simplicity, we neglect small corrections due to $H_{\mathrm{k}}^{\mathrm{M}}$ and logarithmic terms in $k_{\mathrm{B}} T$.) The proposal postulates, as indicated in Fig. 10 by the series of dots, that the annealing changes the situation in such a way that the 
activation enthalpy decreases gradually. At the same time, the pre-exponential relaxation time $\tau_{\infty}$ increases, in qualitative agreement with the observations by D'Anna and Benoit $[12]$.

Proposal (b) has been developed at length elsewhere [11, 15]. Its basic idea is that during the annealing residual impurities (e.g. oxygen atoms) migrate towards the cores of the screw dislocations and modify them in such a way that at the temperature of the internal friction experiments the dislocations do no longer slip on $\{112\}$ but on $\{110\}$ planes, as they do in pure material at low temperatures. The modification of the screw dislocation cores by the incorporation of foreign atoms has been shown to be responsible for the alloy softening in bcc metals $[34,43]$. From Conrad's collection of flow-stress data on impure (by present standards) $\mathrm{Nb}[44]$, the activation enthalpy of $\{110\}$ slip has been deduced as

$$
2 H_{\mathrm{k}}^{\{110\}}=(0.59 \pm 0.02) \mathrm{eV}
$$

[11], in excellent agreement with Eqn (51).

On the basis of the experimental evidence available at present, Proposal (b) is more likely to be the correct one. While Proposal (a) has the attractive feature that it does not invoke the interference of an ill-defined impurity species, in contrast to Proposal (b) it fails to explain why the decrease of $H_{\gamma}$ should stop just at the measured value of $0.59 \mathrm{eV}$, which in Proposal (a) does not have physical significance of its own. As argued elsewhere [11], the idea that the interaction of residual oxygen might modify the screw-dislocation cores and thus be indirectly responsible for the suppression of the 'irreversible' $\gamma$-peak and the appearance of the 'reversible' peak by annealing is indeed supported by the work of Igata and his associates on the influence of oxygen on the $\gamma$-relaxation of $\mathrm{Nb}[45,46]$. A further piece of evidence supporting Proposal (b) is that earlier internal-friction measurements on $\mathrm{Nb}$ containing detectable concentrations of hydrogen atoms, which are known to give rise to alloy softening, showed a peak at $270 \mathrm{~K}(f=1 \mathrm{~Hz})$ with

$$
H_{\gamma}=(0.61 \pm 0.02) \mathrm{eV}, \tau_{0}=1 \times 10^{-12} \mathrm{~s}^{-1}
$$

$[47,48]$ that is fully compatible with Eqns (51) and (52). (Note that in the relaxation process by kink-pair generation in dislocations the activation energy is well-defined, whereas the pre-exponential factor of the relaxation time depends somewhat on the microstructure [42].) 
Acceptance of Proposal (b) implies that the 'reversible peak' of D'Anna and Benoit should be identified with the $\beta$-peak of Chambers [9] and that the criticism by Funk and Schultz [50] of Chamber's concept of an intrinsic $\beta$-relaxation in the refractory bcc metals should be withdrawn.

\section{Discussion and Summary}

\subsection{The flow stress and its interpretation}

As the most impressive feature of the present experiments we consider the striking internal consistency and reproducibility of the flow-stress determinations. This has been made possible by the Ackermann-Mughrabi technique that enabled us to obtain extensive data sets on one single crystal without any change of the crystallographic orientation of the stress axis. Various quantitative cross checks of the interpretation in terms of the theory of kink-pair generation with subsequent kink diffusion could be performed. The data obtained at $\sigma^{*}>$ $\sigma_{\mathrm{M}}$ agreed with those obtained at $\sigma^{*}<\sigma_{\mathrm{M}}$, hence the ansatz Eqn (4) may be considered experimentally verified. The formation energy $2 H_{\mathrm{k}}$ and the height $a$ of kinks in $a_{0}\langle 111\rangle / 2$ screw dislocations determined at effective flow stresses $\sigma^{*}<\hat{\sigma}^{*}$ agree with those determined at effective flow stresses $\sigma^{*}>\hat{\sigma}^{*}$. This shows that the 'upper bend' of the flow-stresstemperature relationship, characterized by the strain-rate-independent effective stress $\hat{\sigma}^{*}$, is not due a change in the dislocations promoting plastic deformation or the mechanism controlling their movement. Rather, the bend is quantitatively accounted for by the transition between two different regimes of the interaction between kinks and anti-kinks as a function of their separation. Moreover, as shown in Sect. 4.4, the 'model-free' determination of the activation enthalpy and the activation volume is fully compatible with the fits to the EI and LT approximation of the kink-pair generation model.

At large kink-kink separations, the interaction between the kinks is mediated by the elastic strain fields of the kinks. Its strength is described by the product $a^{3} b \gamma_{0}=2 \alpha^{2}$, where $a$ is the separation of adjacent Peierls valleys and thus equal to the period of the Peierls potential $U(u), b$ the dislocation strength, and $\gamma_{0}$ the pre-logarithmic factor of the dislocation line tension (equal to $b^{2}$ times a function of the elastic constants and thus computable from 
available information). For $a_{0}\langle 111\rangle / 2$ screw dislocations the experimentally determined $a^{3} b \gamma_{0}$ gives, within experimental error, quantitative agreement with the period $2^{1 / 2} a_{0}$ of the Peierls potential on $\{112\}$ planes. We conclude that in the temperature regime investigated, in which the waviness of the slip-line pattern on the side surfaces indicates frequent cross slip, the $\{112\}$ planes are the elementary slip planes of the screw dislocations, i.e. the planes on which the dislocations slip between the individual cross-slip events. For crystal orientations near the middle of the standard orientation triangle and pre-deformed specimens (this excludes the possibility of anomalous slip $[51,52,53])$ dominance of the $\{110\}$ slip may definitely be excluded in the temperature range investigated. Below the temperature $T=\check{T}$ of the so-called lower bend (not investigated in this work) the dominant slip planes are the $\{110\}$ planes. For a detailed discussion of this transition from the viewpoint of thermodynamics see [34].

At small kink-kink separations, the interaction between neighbouring kinks is no longer independent of the Peierls potential $U(u)$. It may therefore differ somewhat from metal to metal. Nevertheless, without having to specify $U(u)$, the kink-formation energy $H_{\mathrm{k}}$ and the distance $a$ between adjacent Peierls potentials may be derived from the present data with good accuracy . As already alluded to, the numerical values agree well with those obtained at $\sigma^{*}<\hat{\sigma}^{*}$.

(Insert Table 1 about here.) The numerical results from both stress regimes are listed in the first column of Table 1. The data are arranged in three groups. The top group contains the results that may be obtained rather directly from the measurements. (For the meaning of $\tilde{\sigma}$ see Eqn $\left(18_{1}\right)$.) The numerical values, in particular $2 H_{\mathrm{k}}^{\{112\}}$ and $a$, are in good agreement with those derived in a preliminary data analysis [54]. In the last mentioned reference [54] the reader may find a comparison with earlier work of Ackermann et al. [4] and Anglada and Guiu [56]. The entries of the middle group list the information that had to be taken from the literature in addition to the lattice constant $a_{0}$ and the dislocation strength $b$ (in the present case $\left.b=3^{1 / 2} a_{0} / 2\right)$. These values were used to calculate the quantities listed in the bottom group, viz. $U^{\prime \prime}(a)$, defined by Eqn $\left(18_{2}\right)$, and $\beta$, defined by Eqn (19). $U^{\prime \prime}(a)$ is equal to the curvature of the Peierls potential at the bottom of its valleys. $\beta$ characterizes the shape of the Peierls potential since it is independent of its period and its strength. 
Columns 2 and 3 contain the results on Ta and Mo that were obtained by basically the same measuring and data-analysis procedures as used for $\mathrm{Nb}$. The $\beta$ values of all three metals are very close to zero. We recall that the Eshelby potential [27] leads to $\beta=0$. For this potential $H_{\mathrm{kp}}\left(\sigma^{*}\right)$ has been calculated analytically [3, 20]. Nevertheless, for the following reason we have refrained from using this exact result for estimating the Peierls stress $\sigma_{\mathrm{P}}$ from the condition $H_{\mathrm{kp}}\left(\sigma_{\mathrm{P}}\right)=0$, . Whereas the Eshelby potential is symmetric according to the definition (A.6), the Peierls potentials of dislocations with Burgers vector directions $\langle 111\rangle$ and slip planes $\{112\}$ are necessarily asymmetric. This means that in the present case we must distinguish between the Peierls stress in the twinning and in the anti-twinning direction [28]. The appearance of two different Peierls stresses is directly related to the tension-compression asymmetry of the flow stress. Since in the present paper this phenomenon has not been investigated (see Eqn (40)) and since an adequate theoretical treatment requires considerable mathematical effort, we postpone the discussion of the Peierls stress for $\{112\}$ slip. The Peierls stress for $\{110\}$ slip of the $a_{0}\langle 111\rangle / 2$ screw dislocations in the refractory bcc metals may be obtained by extrapolating $\sigma^{*}(T)$ towards $T=0 \mathrm{~K}$.

In the last two columns of Table 1 we have included the results of Brunner [38, 39] on W and $\alpha$-Fe as far as they pertain to $T>\check{T}$. They were obtained by a different technique (stress relaxation after successive tensile deformation) and did not allow us to deduce $D_{\mathrm{k}}, U^{\prime \prime}(a)$ or $\beta$. The compatibility of $a / a_{0}$ with $\{112\}$ slip is complementary to the results shown in the first three columns as it was obtained at plastic strains that exceeded the micro-strain regime only little, in contrast to the cyclic deformation work on $\mathrm{Nb}$, Ta and Mo, which involved high dislocation densities.

The analysis of the present experimental data has been based on Eqn (8). In terms of Fig. 10 this corresponds to $\bar{L}_{\mathrm{k}} \ll x_{\mathrm{k}}$. If this condition was violated, and, hence, Eqn (8) was no longer applicable we should have observed a decrease of the activation energy with decreasing $x_{\mathrm{k}}$, i.e. increasing temperature. In the temperature regime investigated there was no indication for this, hence Eqn (8) was applicable.

The results on $\mathrm{Nb}$ summarized in Table 1 were obtained by deformation in vacuum. To terminate the discussion of the flow-stress measurements we compare them with the results of measurements on three $\mathrm{Nb}$ crystals that were deformed in direct contact with the temperature 
bath. In these measurements the Peierls valley separation $a=(4.98 \pm 0.15) \times 10^{-10} \mathrm{~m}$ was only slightly higher than in Table 1 . The interpretation of the kink diffusivity according to Eqn (34) gave $H_{\mathrm{k}}^{\mathrm{M}}=(15 \pm 10) \mathrm{meV}$ and $D_{0}=\left(0.7_{5} \pm 0.25\right) \times 10^{6} \mathrm{~m}^{2} \mathrm{~s}^{-1}$, i.e. the same results as for the crystals deformed in vacuum. A critical quantity is the formation enthalpy of a kink pair in the limit $\sigma^{*} \rightarrow 0$, which came out as $2 H_{\mathrm{k}}=(0.71 \pm 0.02) \mathrm{eV}$. Its precise value has a strong influence on the numerical value of the product $N_{\mathrm{d}} \bar{L}_{\mathrm{k}}$ of the dislocation density $N_{\mathrm{d}}$ and the mean distance of unsurmountable obstacles to the kink motion, $\bar{L}_{\mathrm{k}}$. In the immersed crystals $N_{\mathrm{d}} \bar{L}_{\mathrm{k}}=(11.5 \pm 2.5) \times 10^{5} \mathrm{~m}^{-1}$ was considerably larger than the "vacuum value' $N_{\mathrm{d}} \bar{L}_{\mathrm{k}}=(3.0 \pm 1.5) \times 10^{5} \mathrm{~m}^{-1}$.

Internal friction measurements $(f=5.7 \mathrm{~Hz})$ showed an exceptionally sharp maximum at $210 \mathrm{~K}$ that is thought to be characteristic of phase transitions in the $\mathrm{Nb}-\mathrm{H}$ system [55]. The direct contribution of the hydride to $\dot{\varepsilon}_{\mathrm{pl}}$ is presumably negligible. The strains accompanying the hydride formation are likely to lead to an increase of $\sigma_{\mathrm{M}}$. This, however, would be difficult to take into account quantitatively, since at $T>210 \mathrm{~K}$ the hydride phase may grow or shrink during the measurements, depending on the pre-history. What is difficult to understand even qualitatively is that $N_{\mathrm{d}} \bar{L}_{\mathrm{k}}$ comes out larger in the immersed samples than in the vacuumdeformed ones. Hydrides on dislocations should have reduced $\bar{L}_{\mathrm{k}}$. The hydride formation might have increased the dislocation density. However, it is hardly conceivable that the density of mobile screw dislocations increases to the extent that the increase of $N_{\mathrm{d}} \bar{L}_{\mathrm{k}}$ by a factor of 4 could be accounted for. A possible explanation is that the increase in $2 H_{\mathrm{k}}$ is an artefact caused by the temperature dependence of the growth or shrinking of hydride particles. The anomalous increase of $N_{\mathrm{d}} \bar{L}_{\mathrm{k}}$ would disappear if the 'true' $2 H_{\mathrm{k}}$ had remained unchanged.

\subsection{Internal friction}

The use of a forced-vibration torsion pendulum enabled D'Anna and Benoit [13] to investigate the $\gamma$-relaxation of $\mathrm{Nb}$ and $\mathrm{Ta}$ over a wider frequency range than was possible before and to determine the activation energy $H_{\gamma}$ rather accurately. In $\mathrm{Nb}$, the activation energy of their 'irreversible' internal-friction peak, $H_{\gamma}=(0.68 \pm 0.03) \mathrm{eV}$, agrees well with $2 H_{\mathrm{k}}$ determined in the present work (see Table 1) that we may consider this as a verification of the Seeger- 


\section{Acknowledgements}

The authors would like to thank Dr. U. Eßmann for his advice in the execution of the vacuum experiments, E. Günther for the skillful fabrication of the vacuum chambers and Dipl. Phys. J. Berger for carrying out the internal friction measurements. One of the authors (U.H.) would like to thank Dr. M. Werner for the introduction in the experimental technique and the theoretical background.

\section{A Appendix - Evaluation of the shape factor $\beta$}

In order to evaluate

$$
\beta:=\int_{u=0}^{a}\left\{u\left[\frac{2 U^{\prime \prime}(a)}{U(u)}\right]^{1 / 2}-\frac{2}{a-u}\right\} \mathrm{d} u,
$$


we introduce the function $F(v)$ by the definition

$$
v \frac{\mathrm{d} F(v)}{\mathrm{d} v}:=u\left[\frac{U^{\prime \prime}(a)}{2 U(u)}\right]^{1 / 2}, \quad v=\frac{u}{a},
$$

and the requirement

$$
F(1 / 2)=0
$$

From (A.2) follows

$$
\left[(1-v) \frac{\mathrm{d} F(v)}{\mathrm{d} v}\right]_{v=1}=\left[v \frac{\mathrm{d} F(v)}{\mathrm{d} v}\right]_{v=0}=1 .
$$

Insertion of (A.2) into (A.1) and integration by parts gives us

$$
\beta=2[v F(v)+\ln (1-v)]_{v=0}^{v=1}-2 \int_{0}^{1} F(v) \mathrm{d} v .
$$

If the potential is 'symmetric', i.e. if

$$
U\left(\frac{a}{2}-u\right)=U\left(\frac{a}{2}+u\right)
$$

$F(v)$ is antisymmetric with respect to $v=a / 2$, hence the integral in (A.5) vanishes and (A.5) then simplifies to

$$
\beta=2 \lim _{v \rightarrow 1}[F(v)+\ln (1-v)] .
$$

Hence the knowledge of $F(v)$ at $v=1$ suffices for the evaluation of $\beta$.

As an example that has enough flexibility to represent a wide range of symmetric periodic potentials we consider

$$
\begin{aligned}
U(u) & =\frac{a^{2} U^{\prime \prime}(a)}{2 \pi^{2}(1+\Lambda)}\left[\sin ^{2}\left(\frac{\pi u}{a}\right)+\frac{\Lambda}{4} \sin ^{2}\left(\frac{2 \pi u}{a}\right)\right] \\
& =\left[\frac{a^{2} U^{\prime \prime}(a)}{2 \pi^{2}}\right] \sin ^{2}\left(\frac{\pi u}{a}\right)\left[1-k^{2} \sin ^{2}\left(\frac{\pi u}{a}\right)\right],
\end{aligned}
$$

where

$$
k^{2}=1-k^{2}=\frac{\Lambda}{(1+\Lambda)}
$$

is a parameter determining the shape of the potential. The case $\Lambda=1, k^{2}=k^{\prime} 2=1 / 2$ corresponds to a potential with a flat maximum at $u=a / 2\left[U^{\prime \prime}(a / 2)=0\right]$. The cases $1 / 2<k^{2}<1$ correspond to potentials with local minima at $u=a / 2(\bmod a)$.

Insertion of (A.9) into (A.2) gives us

$$
\frac{\mathrm{d} F}{\mathrm{~d} v}=\frac{\pi}{\sin \pi v \Delta(\pi v)}
$$


with the abbreviation

$$
\Delta(\pi v):=\left(1-k^{2} \sin ^{2} \pi v\right)^{1 / 2}
$$

The solution of (A.10) satisfying (A.3) is

$$
F(v)=\frac{1}{2} \ln \frac{\Delta(\pi v)-\cos \pi v}{\Delta(\pi v)+\cos \pi v}
$$

hence

$$
\begin{aligned}
\beta & =\lim _{v \rightarrow 1}\left[-\ln \left\{\frac{1-\left(k^{2} / 2\right) \sin ^{2} \pi(1-v)-\cos \pi(1-v)}{2}\right\}+2 \ln (1-v)\right] \\
& =-2\left[\ln \frac{\pi k^{\prime}(1-v)}{2}-\ln (1-v)\right] \\
& =-2 \ln \frac{\pi k^{\prime}}{2} \\
& =+\ln \frac{4(1+\Lambda)}{\pi^{2}} .
\end{aligned}
$$

In the range of $0 \leq k^{2} \leq 1 / 2$ the parameter $\beta$ varies between -0.90 and -0.21 . $\beta$ becomes zero at $\Lambda=1.47$.

\section{References}

[1] A. Kochendörfer and W. Kayser, Arch. Eisenhüttenwesen 39233 (1968).

[2] B. Šesták a. A. Seeger, Z. Metallkd. 69 195, 355 and 425 (1978).

[3] A. Seeger, Z. Metallkd. 72369 (1981).

[4] F. Ackermann, H. Mughrabi and A. Seeger, Acta Metall. 311353 (1983).

[5] A. Einstein, Annalen der Physik 17549 (1905).

[6] A. Seeger, Phil. Mag. 45771 (1953).

[7] H. Mughrabi, Revue de Physique Appliquée 23367 (1988).

[8] A. Seeger and B. Šesták, Scripta Metall. 5875 (1971). 
[9] R.H. Chambers, in Dislocation Relaxations in Body-Centered Cubic Transition Metals, Physical Acoustics, Vol. III A, edited by W.P. Mason (Academic Press, New-York and London, 1966), pp. 123.

[10] A. Seeger, in Selected Topics on Condensed Matter Physics, edited by W.E. Alnaser (University of Bahrain, Bahrain, 1992), pp. 192.

[11] A. Seeger, Phil. Mag. Lett. 8479 (2004).

[12] G. D'Anna and W. Benoit, Mater. Sci. Forum 119-121 165 (1993).

[13] G. D’Anna and W. Benoit, Mater. Sci. Engng A 164191 (1993).

[14] A. Seeger and C. Wüthrich, Nuovo Cimento 33B 38 (1976).

[15] A. Seeger, Mater. Sci. Engng A 37050 (2004).

[16] L. Hollang, M. Hommel, A. Seeger, phys. stat. sol. (a) 160329 (1997).

[17] L. Hollang, D. Brunner, A. Seeger, Mater. Sci. Engng A 319233 (2001).

[18] W. Ulfert and A. Seeger, J. Physique 42 C5-201 (1996).

[19] A. Seeger and P. Schiller, in Physical Acoustics, Principles and Methods Vol. IIIA, edited by W.P. Mason (Academic Press, New York and London, 1966), pp. 361.

[20] A. Seeger, in Dislocations 1984, edited by P. Veyssière, L. Kubin and J. Castaing (CNRS Paris, 1984), pp. 141.

[21] H.A. Kramers, Physica 7284 (1940).

[22] P. Hänggi, P. Talkner and M. Borkovec, Rev. Modern Physics 62251 (1990).

[23] H. O. K. Kirchner, Acta Phys. Austr. 48111 (1978).

[24] M.S. Wu and H.O.K. Kirchner, Phil. Mag. 842485 (2004).

[25] H.-M. Hauser, Diplomarbeit (Universität Stuttgart, 1995)

[26] A. Seeger, L. Schimmele and H.-M. Hauser, to be published

[27] J.D. Eshelby, Proc. Roy. Soc. Lond. A266, 222 (1962) 
[28] A. Seeger, Z. Metallkd. 93760 (2002).

[29] J. Fuss, Diplomarbeit (Universität Stuttgart, 1975)

[30] K. Schulze, J. Fuss, H. Schultz, S. Hofmann, Z. Metallkd. 67737 (1976)

[31] A. Luft, J. Richter, K. Schlaubitz, Ch. Loose and Ch. Mühlhaus, Mater. Sci. Engng 20 $113(1975)$.

[32] Y.T. Chou and G.T. Sha, Metall. Trans. A 32857 (1972).

[33] P.E. Armstrong, J.M. Dickinson and H.L. Brown, Trans. Metal. Soc. AIME 2361404 (1966).

[34] A. Seeger, J. Physique IV 5, C7-45 (1995).

[35] D.M. Barnett, R.J. Asaro, S.D. Gavazza, D.J. Bacon, and R.O. Scattergood, J. Phys. F 2654 (1972).

[36] M. Werner, phys. stat. sol. (a) 10463 (1987).

[37] M. Werner and A. Seeger, in: Proc. 8th International Conference on the Strength of Metalls and Alloys, Tampere, Finnland, ed. by P.O. Kettunen, T.K. Lepistö, M.E. Lehtonen (Pergamon Press, Oxford, 1988), pp. 173.

[38] D. Brunner, Mater. Trans. JIM 41152 (2000).

[39] D. Brunner and J. Diehl, phys. stat. sol.(a) 104355 (1991).

[40] G. Schoeck, phys. stat. sol. 8499 (1965).

[41] A. Seeger, Phil. Mag. Lett. 83107 (2003).

[42] A. Seeger, Journal de Physique 42 C5-201 (1981).

[43] A. Seeger and W. Wasserbäch, Matériaux \& Techniques 91, 51 (2003).

[44] H. Conrad, in: The Relation between the Structure and Mechanical Properties of Metals, National Physical Laboratory Symposium 15 (London: Her Majestiy's Stationary Office, 1963), pp. 467. 
[45] N. Igata, H. Murakami, H. Hiramatsu, and N. Masamura, in: Internal Friction and Ultrasonic Attenuation in Solids, edited by R.R. Hasiguti and N. Mikoshiba (University of Tokto Press, 1977), pp. 677.

[46] N. Igata, H. Murakami, H. Hiramatsu, and N. Masamura, Scripta metall. 14463 (1980).

[47] F. de Lima and W. Benoit, J. Physique 42 C5-85 (1981).

[48] F. De Lima and W. Benoit: pys. sat. sl.(a) 67565 (1981).

[49] H.G. Schmid, Mater. Sci. Engng 59 L7 (1983).

[50] G. Funk and H. Schultz, Z. Metallkd. 7631 (1985).

[51] M.S. Duesbery and A. Foxall, Phil. Mag. 2071 (1969).

[52] A. Seeger, Mater. Sci. Engng A 319-321 254 (2001).

[53] A. Seeger and W. Wasserbäch, phys. stat. sol. (a) 18027 (2002).

[54] U. Holzwarth and A. Seeger, in: Proc. 9th International Conference on the Strength of Metalls and Alloys, ed. by D.G. Brandon, R. Chaim and A. Rosen; (Freund Publishing House Ltd, London 1991) pp. 577.

[55] O. Buck, D.O. Thompson, C. Wert, Scripta Metall. 81061 (1974)

[56] M. Anglada and F. Guiu, Phil. Mag. 46881 (1981).

[57] J. Baur, W. Benoit, and H. Schultz, Acta metall. 371159 (1989).

[58] B.L. Mordike and P. Haasen, Phil. Mag. 7459 (1962) 


\begin{tabular}{|c|c|c|c|c|c|}
\hline Material & $\mathrm{Nb}$ & $\mathrm{Ta}[36,37]$ & $\mathrm{Mo}[16]$ & $\mathrm{W}[38]$ & $\alpha$-Fe $[39]$ \\
\hline \hline $\begin{array}{c}\text { experimental } \\
\text { technique }\end{array}$ & \multicolumn{3}{|c|}{ Ackermann-Mugrabi technique } & \multicolumn{2}{|c|}{$\begin{array}{c}\text { successive tensile } \\
\text { deformations }\end{array}$} \\
\hline $2 H_{\mathrm{k}}[\mathrm{eV}]$ & $0.68 \pm 0.02$ & $0.98 \pm 0.02$ & $1.27 \pm 0.02$ & 1.75 & 0.93 \\
$H_{\mathrm{k}}^{\mathrm{M}}[\mathrm{meV}]$ & $15 \pm 10$ & - & $30 \pm 20$ & - & - \\
$D_{0}\left[10^{-6} \mathrm{~m}^{2} \mathrm{~s}^{-1}\right]$ & $0.9 \pm 0.3$ & - & $4.8 \pm 0.2$ & - & - \\
$a\left[10^{-10} \mathrm{~m}\right]$ & $4.78 \pm 0.14$ & $4.67 \pm 0.11$ & $4.50 \pm 0.13$ & 4.5 & 3.9 \\
$a / a_{0}$ & $1.45 \pm 0.04$ & $1.41 \pm 0.03$ & $1.37 \pm 0.04$ & 1.4 & 1.4 \\
$\hat{\sigma}^{*}[\mathrm{MPa}]$ & $93 \pm 5$ & $80 \pm 3$ & $180 \pm 1$ & 150 & - \\
$\tilde{\sigma}[\mathrm{GPa}]$ & $7.1 \pm 0.1$ & $5.8 \pm 0.2$ & $14.8 \pm 0.2$ & - & - \\
\hline$\gamma_{0}\left[10^{-10} \mathrm{~N}\right]$ & $3.4[35]$ & $11.8[49]$ & $7.88[32]$ & $9.55[32]$ & $12.7[49]$ \\
$\ln \left(r_{\infty} / r_{0}\right)$ & $2.55[25]$ & $2.50[25]$ & $2.80[25]$ & - & - \\
\hline$U^{\prime \prime}(a)[\mathrm{GPa}]$ & $2.0 \pm 0.1$ & $1.7 \pm 0.1$ & $4.45 \pm 0.24$ & - & - \\
$\beta$ & $0.06 \pm 0.05$ & $0.04 \pm 0.04$ & $0.005 \pm 0.005$ & - & - \\
\hline
\end{tabular}

Table 1: Compilation of the kink-pair formation energy $2 H_{\mathrm{k}}$, the kink migration energy and pre-exponential factor, $H_{\mathrm{k}}^{\mathrm{M}}$ and $D_{0}$, the kink height $a$, the 'hump' stress $\hat{\sigma}^{*}$, and the parameter $\tilde{\sigma}$ defined in Eqn (26) as derived from flow-stress measurements on $\mathrm{Nb}$ (this work), Ta, Mo, $\mathrm{W}$, and $\alpha$-Fe. The values for $\gamma_{0}$ and $\ln \left(r_{\infty} / r_{0}\right)$ are literature data used to calculate the quantities $U^{\prime \prime}(a)$ and $\beta$ from Eqns (27) and (18). 


\section{Figure Captions}

Figure 1: Double logarithmic plot of $a b \sigma^{*} / \gamma_{0}$ versus $\left(k_{\mathrm{B}} T / D_{\mathrm{k}}\right) \times\left(a / m_{\mathrm{k}} \gamma_{0}\right)^{1 / 2}$ illustrating the regimes in which the kink-kink interaction can be described by the line-tension approximation or the elastic-interaction approximation. The range of validity of the transition-state theory is separated by the bold lines from the regime of kink-diffusion control.

Figure 2: Longitudinal cut of a vacuum chamber protecting the Nb crystals from contamination up-take by direct exposure to the temperature bath. The extensions on the hatched lids of the chamber housing the gripping part of the $\mathrm{Nb}$ crystals are very thin $(0.2 \mathrm{~mm})$; they are represented by a line, visible between disc (lid) and the spacer.

Figure 3: Temperature and strain rate dependence of the effective stress $\sigma^{*}\left(T, \dot{\varepsilon}_{\mathrm{pl}}\right)$ determined on the single crystal $\mathrm{Nb} 95 / 1$ after pre-deformation at $370 \mathrm{~K}$. Above $350 \mathrm{~K}, \sigma^{*}\left(T, \dot{\varepsilon}_{\mathrm{pl}}\right) \equiv 0$ for all strain rates investigated.

In the regime of the line-tension approximation (LT) the lines are fitted to the experimental data using Eqn (39). The transition between the regimes of the line-tension and the elastic interaction approximation at a strain-rate independent stress $\hat{\sigma}^{*} \approx 93 \mathrm{MPa}$ is indicated. In the transition-state approximation regime $\left(\sigma^{*} \geq 25 \mathrm{MPa}\right)$ the fits were based on Eqn (42), at higher temperatures with non-negligible effects of kink diffusion $\left(\sigma^{*}<25 \mathrm{MPa}\right)$ on Eqn (33).

Figure 4: Determination of the knee temperatures $T_{\mathrm{K}}$. The extrapolation of the straight lines to $\sigma^{* 1 / 2}=0$ determines the knee temperatures for each $\dot{\varepsilon}_{\mathrm{pl}}$. The extrapolation to zero temperature gives $A T_{\mathrm{K}}=(24 \pm 0.5) \mathrm{MPa}^{1 / 2}$.

Figure 5: Determination of $2 H_{\mathrm{k}}$ and $\dot{\varepsilon}_{0}$ according to Eqn (37). 
Figure 6: Arrhenius plot of the kink diffusivity $D_{\mathrm{k}}$. The open squares denote preliminary data calculated from triples $\left(\dot{\varepsilon}_{\mathrm{pl}}, \sigma^{*}, T\right)$ making use of $H_{\mathrm{k}}, \alpha, \dot{\varepsilon}_{0}$ as determined in the range of validity of the transition-state theory. The filled circles result from fits of $D_{\mathrm{k}}(T)$ to sets of $\sigma^{*}\left(T=\right.$ const,$\left.\dot{\varepsilon}_{\mathrm{pl}}\right)$ at temperatures at which $\sigma^{*}$ has been measured for 10 different $\dot{\varepsilon}_{\mathrm{pl}}$-values. The slope yields $H_{\mathrm{k}}^{\mathrm{M}}=(15 \pm 10) \mathrm{meV}$.

Figure 7: Plot of $k_{\mathrm{B}} T \ln \left(\dot{\varepsilon}_{0} / \dot{\varepsilon}_{\mathrm{pl}}\right)$ versus $\sigma^{*}\left[1+\ln \left(\tilde{\sigma} / \sigma^{*}\right)\right]$ for $\tilde{\sigma}=7.1 \mathrm{GPa}$. The extrapolation of the straight-line fit to $\sigma^{*}=0$ yields $2 H_{\mathrm{k}}=\left(0.68_{5} \pm 0.01_{5}\right) \mathrm{eV}$. The slope of the line is $(9.13 \pm 0.13) \times 10^{-29} \mathrm{~m}^{3}=3.9 b^{3}$.

Figure 8: Stress dependence of the kink-pair formation enthalpy $H_{\mathrm{kp}}\left(\sigma^{*}\right)$. The experimental data were obtained by means of Eqn (48). The full line is a fit of Eqn (11) with $2 H_{\mathrm{k}}=0.69 \mathrm{eV}$, $a=4.8 \times 10^{-10} \mathrm{~m}$. The dash-dotted line a fit of Eqn (21) using the same values for $2 H_{\mathrm{k}}$ and $a$ together with $\hat{\sigma}=7.1 \mathrm{GPa}$ and $U^{\prime \prime}(a)=2.0 \mathrm{GPa}$. The transition between the EI and the LT approximation at $\hat{\sigma}^{*} \approx 93 \mathrm{MPa}$ is indicated.

Figure 9: Stress dependence of the activation volume $V_{\mathrm{kp}}\left(\sigma^{*}\right)$. The experimental data were obtained by means of Eqn (47). The transition between the LT and the EI approximation regimes at $\hat{\sigma}^{*}$ is indicated. The ratio of the extrapolations of $V_{\mathrm{kp}}^{\mathrm{LT}}\left(\sigma^{*}\right)$ and $V_{\mathrm{kp}}^{\mathrm{EI}}\left(\sigma^{*}\right)$ towards $\left(\hat{\sigma}^{*}\right)$ is $1.61 \pm 0.05$ (cf. Eqn $\left.(28)\right)$.

Figure 10: Schematic plot of $\ln L_{\mathrm{k}}$ versus $1 / k_{\mathrm{B}} T$. Increasing $\bar{L}_{\mathrm{k}}$ during annealing (see dots) causes the activation energy $H_{\gamma}$ to decrease gradually from $2 H_{\mathrm{k}}$ to $H_{\mathrm{k}}$. 